OPEN ACCESS

Edited by:

Lei Deng,

Central South University, China

Reviewed by:

Deliang Cao,

Southern Illinois University

Carbondale, United States

Changzheng Liu,

Institute of Pathogen Biology

(CAMS), China

${ }^{*}$ Correspondence:

Xiequn Xu

xxq75@163.com

Ziwen Liu

liuziwenpumch@163.com

Xiaobin Li

xbli2008@sina.com

†These authors have contributed equally to this work

Specialty section: This article was submitted to

Molecular Medicine,

a section of the journal

Frontiers in Cell and Developmental

Biology

Received: 14 September 2020

Accepted: 11 December 2020

Published: 21 January 2021

Citation:

Wu M, Li S, Han J, Liu R, Yuan H,

XU X, Li X and LiU Z (2021)

Progression Risk Assessment of

Post-surgical Papillary Thyroid

Carcinoma Based on Circular

RNA-Associated Competing

Endogenous RNA Mechanisms.

Front. Cell Dev. Biol. 8:606327.

doi: 10.3389/fcell.2020.606327

\section{Progression Risk Assessment of Post-surgical Papillary Thyroid Carcinoma Based on Circular RNA-Associated Competing Endogenous RNA Mechanisms}

\author{
Mengwei $\mathrm{Wu}^{1+}$, Shuo $\mathrm{Li}^{1 \dagger}$, Jiashu Han ${ }^{1,2+}$, Rui Liu ${ }^{1}$, Hongwei Yuan ${ }^{1}$, Xiequn Xu ${ }^{1 *}$, \\ Xiaobin $\mathrm{Li}^{1 *}$ and Ziwen Liu ${ }^{1 *}$ \\ ${ }^{1}$ Department of General Surgery, Peking Union Medical College Hospital, Chinese Academy of Medical Sciences \& Peking \\ Union Medical College, Beijing, China, ${ }^{2}$ MD Program, Chinese Academy of Medical Sciences \& Peking Union Medical \\ College, Beijing, China
}

Background: Accurate risk assessment of post-surgical progression in papillary thyroid carcinoma (PTC) patients is critical. Exploring key differentially expressed mRNAs (DE-mRNAs) regulated by differentially expressed circular RNAs (circRNAs) via the ceRNA mechanism could help establish a novel assessment tool.

Methods: ceRNA network was established based on differentially expressed RNAs and correlation analysis. DE-mRNAs within the ceRNA network associated with progression-free interval (PFI) of PTC were identified to construct a prognostic ceRNA regulatory subnetwork. least absolute shrinkage and selection operator (LASSO)-Cox regression was applied to identify hub DE-mRNAs and establish a novel DE-mRNA signature in predicting PFI of PTC.

Results: Six hub DE-mRNAs, namely, CLCNKB, FXBO27, FXYD6, RIMS2, SPC24, and CDKN2A, were identified to be most significantly related to the PFI of PTC, and a prognostic DE-mRNA signature was proposed. A nomogram incorporating the DE-mRNA signature and clinical parameters was established to improve the progression risk assessment in post-surgical PTC, which was superior to the American Thyroid Association risk stratification system and distant Metastasis, patient Age, Completeness of resection, local Invasion, and tumor Size (MACIS) score American Joint Committee on Cancer staging system.

Conclusions: Based on the circRNA-associated ceRNA RNA mechanism, a DE-mRNA signature and prognostic nomogram was established, which may improve the progression risk assessment in post-surgical PTC.

Keywords: nomogram, papillary thyroid carcinoma, ceRNA, circRNA, progression-free interval

\section{INTRODUCTION}

Thyroid cancer, originating from thyroid follicular epithelial cells, is the most common malignancy of the endocrine system (La Vecchia et al., 2015). Thyroid cancer is currently the eighth most common malignancy around the world (Antonelli and La Motta, 2017), and its incidence is rapidly increasing worldwide. There are four main types of thyroid cancer, including papillary thyroid 
carcinoma (PTC), follicular thyroid carcinoma, anaplastic thyroid carcinoma, and medullary thyroid carcinoma. Of these, PTC accounts for more than $80 \%$ of all thyroid cancer cases. Among all types of thyroid cancer, PTC has the highest incidence with a higher proportion of young patients. Genetic mutations and environmental exposures are the major risk factors for PTC in which BRAF V600E is the most common mutation. The primary treatment for PTC is thyroidectomy. Most PTCs grow slowly, and $90 \%$ of patients have a relatively good prognosis after treatment, with a low post-operative recurrence rate $<10 \%$ (Haugen et al., 2016). However, a small percentage of patients experience a high risk of recurrence and metastasis after surgery, resulting in a poor prognosis.

The molecular mechanisms leading to PTC recurrence remain unclear. Moreover, there is a lack of satisfactory assessment tools to precisely evaluate the post-surgical risk of recurrence in PTC. The American Thyroid Association (ATA) currently recommends the use of American Joint Committee on Cancer (AJCC) staging system and distant Metastasis, patient Age, Completeness of resection, local Invasion, and tumor Size (MACIS) score to predict mortality after PTC and proposed the risk stratification system for risk assessment of postsurgical progression in PTC (Haugen et al., 2016). However, the accuracy of currently available assessment tools is inadequate to meet the requirements of clinical work. The challenge of treating PTC lies in balancing side effects and treatment benefits (Cabanillas et al., 2016). Patients with low- and high-risk PTC should be differentiated to adopt different treatment strategies. Patients with high risk of recurrence require more extensive surgical resection and adjuvant radioactive iodine therapy to improve post-surgical prognosis and prevent recurrence. Secondary surgery for recurrence can result in additional surgical trauma with a higher risk of recurrent laryngeal nerve injury. Alternatively, PTC patients with low risk of recurrence should receive a more conservative treatment to reduce surgical trauma and improve quality of life. Unnecessary post-surgical radioactive iodine therapy and thyroid-stimulating hormone (TSH) suppression therapy should also be avoided. Longterm subclinical hyperthyroidism caused by a high dose of TSH suppression can lead to multiple potential side effects, including osteoporosis, atrial fibrillation, heart discomfort, and an increased risk of fractures and heart disease in elderly patients (Biondi and Cooper, 2018). Unnecessary radioactive iodine therapy also increases the risk of developing other cancers. Therefore, more accurate assessment tools to assess the risk of progression in post-surgical PTC patients are urgently required.

Non-coding RNAs such as microRNAs (miRNAs), long non-coding RNAs, and circular RNAs (circRNAs) are major components of the human transcriptome. circRNAs were first discovered in human cells in 1986 and are critical in the pathophysiology of several human diseases including cancer (Kos et al., 1986). circRNAs are products of a reverse splicing mechanism and are resistant to RNase R (Chen and Yang, 2015). circRNAs contain a single-stranded covalent closedloop structure that have neither a $5^{\prime}-3^{\prime}$ polarity nor a polyadenylated tail. circRNAs are primarily located in the cytoplasm and regulate gene expression by acting as an
miRNA sponge at the transcriptional or post-transcriptional stage, affecting protein translation and thus the regulation of cellular processes. According to the competitive endogenous RNA (ceRNA) hypothesis, non-coding RNAs such as circRNAs can positively regulate mRNA expression by competitive binding of its shared miRNAs (Salmena et al., 2011). Recent studies further reveal that several circRNAs differentially expressed in cancers can exert a tumor-promoting or tumorsuppressing role via ceRNA mechanism, thus regulating apoptosis, proliferation, invasion, and metastasis (Zhong et al., 2018; Liu et al., 2020). mRNAs regulated by differentially expressed circRNAs (DE-circRNAs) may be more likely to play a functional role in cancer. Particularly in PTC, the upregulated SOX2 by circ_0005273/hsa-miR-1183 axis promotes the proliferation, invasion, and metastasis of PTC (Zhang et al., 2020). Transforming growth factor $\alpha$, upregulated by hsa-circ0060060/hsa-miR-144-3p axis in PTC, was identified to promote the proliferation and autophagy of PTC and was associated with poor prognosis (Liu et al., 2018). The establishment of circRNAmiRNA-mRNA regulatory networks in PTC may provide key targets and novel regulatory pathways in understanding the progression of PTC.

The primary objective of this study was to explore key differentially expressed mRNAs (DE-mRNAs) regulated by DEcircRNA-associated ceRNA regulatory mechanism in PTC to elucidate the post-surgical progression of PTC and establish a novel assessment tool.

\section{MATERIALS AND METHODS}

\section{Identification of DE-circRNAs, DE-miRNAs, and DE-mRNAs in PTC}

Expression data of circRNAs and related clinical data for PTC were downloaded from the GSE93522 dataset based on GPL19978 platform (Peng et al., 2017). An annotation file provided by the manufacturers was used to match the probes with corresponding circRNA IDs. The median ranking value was used to determine the expression value if multiple probes matched a single circRNA ID. The 'LIMMA' $\mathrm{R}$ package was used to identify DE-circRNAs between cancer and normal tissues (Ritchie et al., 2015). The cutoff value was set at $|\log 2 \mathrm{FC}|>1$ and $P<0.05$. The basic characteristics of DEcircRNAs were obtained from circBase (https://www.circbase. org/), and the corresponding structures were analyzed using the Cancer-Specific circRNA Database (CSCD) (https://gb.whu.edu. $\mathrm{cn} / \mathrm{CSCD} / \# /)$.

Normalized RNA sequencing data in the form of millions of transcripts (TPM) for miRNA and mRNA and related clinical data of post-surgical PTC were downloaded from The Cancer Genome Atlas (TCGA) (https://portal.gdc.cancer.gov/, up to July 1, 2020). TCGA-Thyroid Cancer (THCA) dataset originally included 507 cases, 510 tumor samples, and 58 normal tissue samples. A total of 495 cases of PTC with matching tumor tissue and clinical information, and 58 normal samples of thyroid tissue were eventually included in the current analysis following the removal of five cases without matching tumor 
samples, two cases with poorly differentiated oncolytic carcinoma or follicular carcinoma, five cases with history of neoadjuvant therapy, and eight samples of metastases. The 'LIMMA' R package was used to identify DE-miRNAs and DE-mRNAs between cancer and normal tissues with a cutoff value set at $|\log 2 \mathrm{FC}|$ $>1$ and a false discovery rate (FDR) $<0.05$ (Ritchie et al., 2015). GEPIA (http://gepia.cancer-pku.cn) incorporating RNA sequencing expression data of tumors and normal samples from TCGA and the Genotype-Tissue Expression projects was used to validate the differential expression level of a specific DEmRNA (Tang et al., 2017). Data of copy number alterations and mutation were downloaded from Cbioportal (http://www. cbioportal.org/).

\section{Construction of the circRNA-miRNA-mRNA Network}

CIRCinteractome is a computational tool that enables the prediction and mapping of binding sites for RNA-binding proteins and miRNAs on reported circRNAs (Dudekula et al., 2016). circRNA-miRNA relationships were predicted using CIRCinteractome. miRWalk is an open-source platform that provides predicted and validated miRNA-binding sites with a machine learning algorithm, incorporating data from TargetScan (conserved site context scores, version 7.1), miRDB (release 5.0), and validated information from miRTarBase (version 7.0) (Sticht et al., 2018). miRNA-mRNA relationships were predicted using miRWalk 3.0 with score $\geq 0.95$. Based on differential expression data, only relationship pairs with negative correlation were retained. The correlation between DE-miRNA and DEmRNA expression with potential ceRNA regulatory relationships was further analyzed using Pearson correlation coefficient in TCGA-THCA dataset. Only miRNA-mRNA relationships with $r$ $<-0.2$ and $P<0.05$ were retained in the final circRNA-miRNAmRNA network. To construct a ceRNA regulatory subnetwork associated with progression-free interval (PFI) of PTC, univariate Cox regression analysis was performed based on the DEmRNAs involved. Only DE-mRNAs associated with PFI of PTC and the corresponding circRNA-miRNA and miRNA-mRNA pairs were retained in the PFI-related subnetwork. The ceRNA network was visualized using Cytoscape v.3.8.0 (https://www. cytoscape.org/). The Sankey diagram that represented the ceRNA regulatory relationship was drafted using Origin 2020 (https:// www.originlab.com/).

\section{Bioinformatics Analysis of the circRNA-miRNA-mRNA Network}

To investigate the underlying biological function of ceRNA regulatory relationships in PTC, DAVID (https://david.ncifcrf. gov/) was employed to explore enriched biological processes, cellular components, molecular functions, and significantly relevant signal pathways of DE-mRNAs involved, using default parameters (Huang da et al., 2009). $P<0.05$ was regarded statistically significant. The results were visualized using the OmicShare tools, a free online platform for data analysis (http:// www.omicshare.com/tools).

\section{Identification of PFI-Related Hub DE-mRNAs and Establishment of the Prognostic DE-mRNA Signature}

In this study, PFI was selected as the main endpoint (https://wiki. nci.nih.gov/plugins/servlet/mobile\#content/view/24279961).

DE-mRNAs associated with PFI were identified based on TCGA-THCA dataset using a univariate Cox proportional hazards regression model. Normalized gene expression data were transformed on the base-2 logarithm for further survival analysis. DE-mRNAs with $P<0.05$ were considered statistically significant for further analysis. Only cases with follow-up $>30$ days were included for survival analysis. The 492 eligible TCGA cases were subsequently randomly divided into a training dataset and a validation dataset in a 7:3 ratio. Least absolute shrinkage and selection operator (LASSO) penalized Cox regression analysis was performed to select prognostic hub DE-mRNAs related to PFI and constructed a prognostic DE-mRNA signature in patients with PTC based on a linear combination of the regression coefficient derived from LASSO-Cox regression model coefficients $(\beta)$ multiplied with its normalized mRNA expression level in the training dataset. Patients were divided into the high-risk and the low-risk group based on the optimal cutoff value determined by X-Tile (Camp et al., 2004). Performance of the prognostic DE-mRNA signature was assessed using Harrell's concordance index (C-index), area under the curve (AUC) of the receiver operating characteristic (ROC) curve, and KaplanMeier analysis. The validation dataset and TCGA-THCA dataset were further utilized for validation.

\section{Gene Set Enrichment Analysis}

Gene Set Enrichment Analysis (GSEA) was performed to compare the molecular alteration in the high-risk group and the low-risk group against previously annotated gene sets (Subramanian et al., 2005). Samples from TCGA-THCA dataset were divided into the high-risk group and the low-risk group according to the optimal cutoff value determined by X-Tile. Thereafter, GSEA was run on javaGSEA v4.0.3 based on the Molecular Signatures Database v7.1. H: Hallmark gene sets, C2: curated gene sets and C5: gene otology gene sets were searched to identify enriched Kyoto Encyclopedia of Genes and Genomes (KEGG) pathways, biological processes, cellular components, molecular functions, and specific well-defined biological states or processes associated with poorer survival of the high-risk group. Gene sets with $|\mathrm{NES}|>1$ and FDR $<0.25$ were considered statistically significant.

\section{Validation of the Independent Prognostic Role of the DE-mRNA Signature}

To validate the independent prognostic role of the DE-mRNA signature, univariate and multivariate Cox regression analyses were performed on the prognostic DE-mRNA signature and clinical parameters, including age; PFI status; mutational status of BRAF V600E, RAS, RET, NTRK1, and TERT; sex; histological subtype; T stage; $\mathrm{N}$ stage; $\mathrm{M}$ stage; AJCC stage; residual tumor status; extrathyroidal extension; multifocality; and anatomic site in TCGA dataset. Parameters with $P<0.25$ in univariate analysis 
were further included in the multivariate Cox regression analysis. $P<0.05$ was considered statistically significant.

\section{Building and Validation of a Prognostic Nomogram}

Following a test for collinearity, independent prognostic parameters and relevant clinical parameters were included to construct a prognostic nomogram to predict 1-, 2-, 3-, 4-, and 5 -year PFI of PTC patients in the entire TCGA dataset with the stepwise Cox regression model. Discrimination of the nomogram was assessed with the C-index calculated by a bootstrap method with 1,000 resamples. The predicted progression-free survival of nomogram against observed survival rates was plotted using the calibration curve. The performance of the prognostic nomogram was further assessed with ROC curves and Kaplan-Meier analysis. The ATA risk stratification system, MACIS score, and AJCC staging system were used as references for comparison of AUCs. Information of ATA risk stratification and MACIS score for each case was obtained from the official TCGA publication (Cancer Genome Atlas Research, 2014).

\section{Statistical Analysis}

Statistical analysis was conducted using R software v3.4.3 (https:// www.r-project.org/), and GraphPad Prism v8.01 (https://www. graphpad.com/). Categorical variables were analyzed with $\chi^{2}$ test or Fisher exact test. Continuous variables of two groups were analyzed with Student $t$-test. Multiple groups of continuous variables were analyzed with a one-way analysis of variance test. DE-mRNAs associated with progression-free survival were identified based on univariate and multivariate Cox regression analysis. Hazard ratio (HR) and 95\% confidence interval (CI) were calculated. Correlation between two variables was analyzed with Pearson correlation coefficient. ROC curves were analyzed using the "timeROC" R package, and the AUCs were compared with the method described by Blanche et al. (2013). A two-tailed $P<0.05$ was considered statistically significant if there was no special statement.

\section{RESULTS}

\section{Identification of DE-circRNAs, DE-miRNAs, and DE-mRNAs in PTC}

Workflow of the current study is shown in Figure 1A. DEcircRNAs, miRNAs, and mRNAs between PTC and normal thyroid tissues were identified after normalization of expression data. A total of 138 DE-circRNAs were obtained from GSE93522, of which 117 DE-circRNAs were upregulated and 21 DEcircRNAs were downregulated (Supplementary Table 1). DEmiRNAs and DE-mRNAs were obtained from TCGA-THCA dataset. Altogether, 113 DE-miRNAs and 2,252 DE-mRNAs were identified (Supplementary Tables 2, 3). Among these, $32 \mathrm{DE}$ miRNAs and 905 DE-mRNAs were upregulated, and 81 DEmiRNAs and 1347 DE-mRNAs were downregulated.

\section{Prediction of Potential Circular RNA-Associated ceRNA Regulatory Relationships and Functional Enrichment Analysis}

circRNA-miRNA relationships were predicted using Circular RNA Interactome. miRNA-mRNA relationships were predicted using mirWalk 3.0. Based on the targeted relationship combined with differential expression data, 93 potential circRNA-miRNA pairs and 1,105 miRNA-mRNA pairs were identified, which involved 54 DE-circRNAs, 14 DE-miRNAs, and 698 DE-mRNAs (Figures 1B-D and Supplementary Tables 4, 5).

To investigate the underlying biological function of ceRNA regulatory relationships in PTC, functional enrichment analysis was performed based on the 698 DE-mRNAs involved (Figures 2A-D and Supplementary Table 6). Biological processes associated with malignant phenotype were significantly enriched, which included regulation of cell adhesion, extracellular matrix disassembly and organization, positive regulation of cell proliferation, and angiogenesis. Processes associated with intracellular signal transduction were also significantly enriched, particularly the positive regulation of the mitogen-activated protein kinase cascade. In terms of KEGG pathways, DE-mRNAs were significantly associated with transcriptional misregulation and miRNAs in cancer. Multiple tumor-associated signaling pathways were also enriched, including $\mathrm{p} 53$ and PI3K-Akt signaling pathways.

\section{Construction of the circRNA-miRNA-mRNA Network}

The correlation between DE-miRNA and DE-mRNA expression with potential ceRNA regulatory relationships in PTC was further analyzed using Pearson correlation coefficient. Only miRNA-mRNA relationships with negatively correlated expression were retained. Correlation analysis of miRNAs and potential target mRNAs in TCGA-THCA dataset are shown in Supplementary Table 7. A ceRNA network was constructed, which included 25 DE-circRNAs, 6 DE-miRNAs, and 150 DE-mRNAs based on 32 circRNA-miRNA pairs and 153 miRNA-mRNA pairs (Figure 3).

To construct a ceRNA regulatory subnetwork associated with PFI in PTC, univariate Cox regression analysis was performed based on the 150 DE-miRNAs involved. A total of 27 DE-mRNAs were identified to be associated with PFI of PTC. Following retention of the corresponding regulatory circRNA-miRNA and miRNA-mRNA pairs, a ceRNA regulatory subnetwork associated with PFI in PTC was constructed. This included 11 DE-circRNAs, 3 DE-miRNAs and 27 DE-mRNAs based on 11 circRNA-miRNA pairs, and 27 miRNA-mRNA pairs (Figure 4A). hsa_circ_0003645, hsa_circ_0089153, hsa_circ_0005699, hsa_circ_0007146, hsa_circ_0038718, hsa_circ_0001658, hsa_circ_0008784, hsa_circ_0000965, hsa_circ_0001917, hsa_circ_0008354, and hsa_circ_0049271 were DE-circRNAs within the PFI-related subnetwork. The basic characteristics of the $11 \mathrm{DE}$-circRNAs are shown in Table 1. The corresponding structures of DE-circRNAs were analyzed using the CSCD and shown in Figure 4B. 
A

B

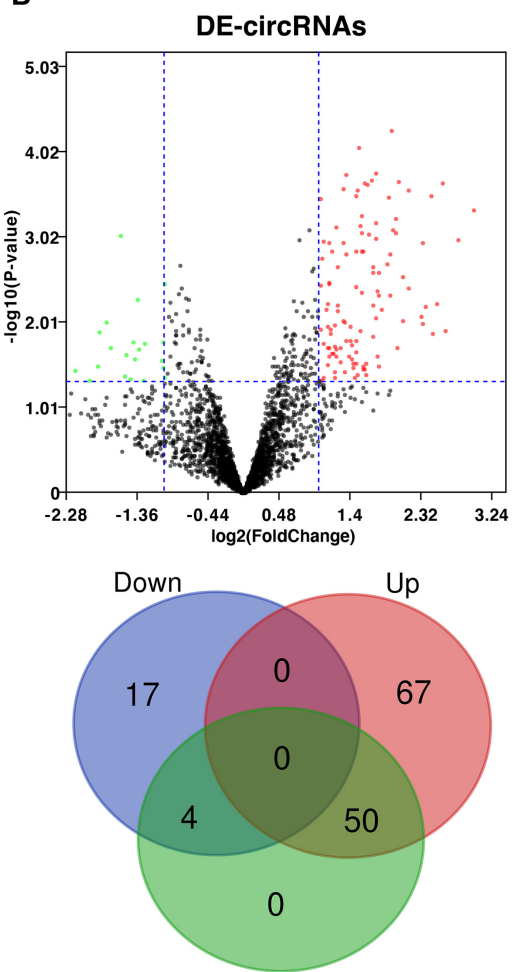

ce targets

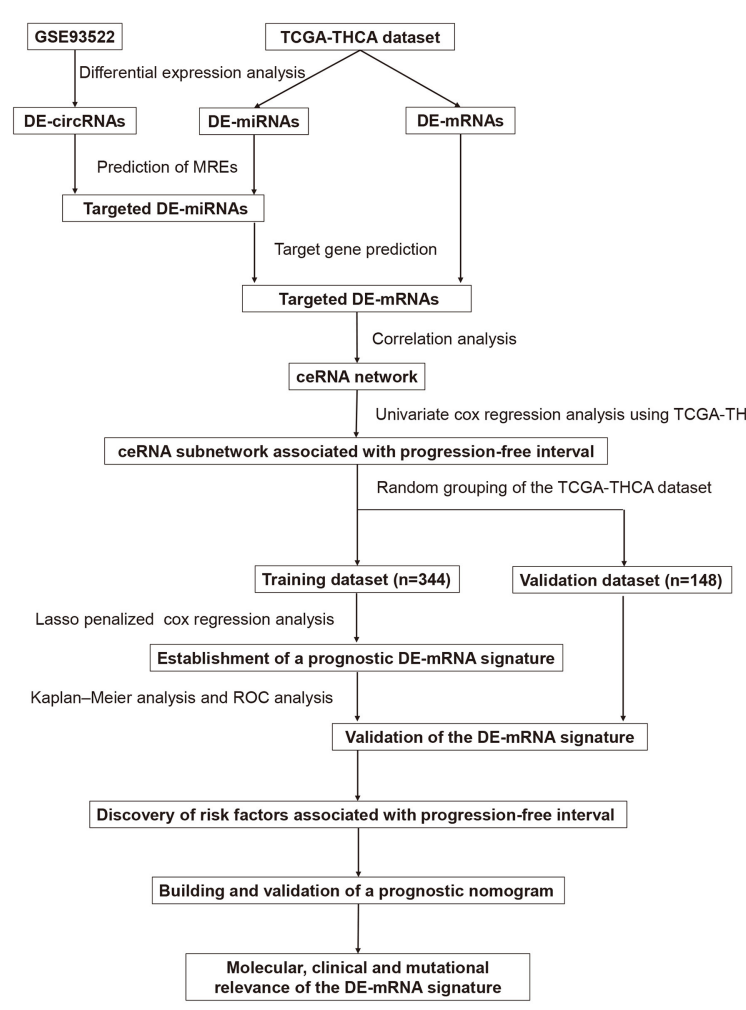

C

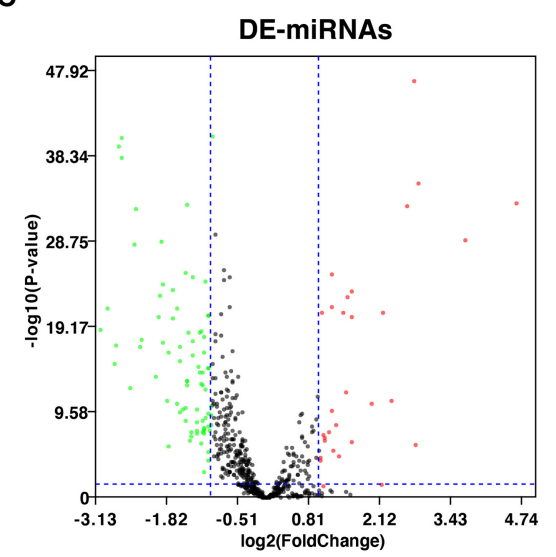

D
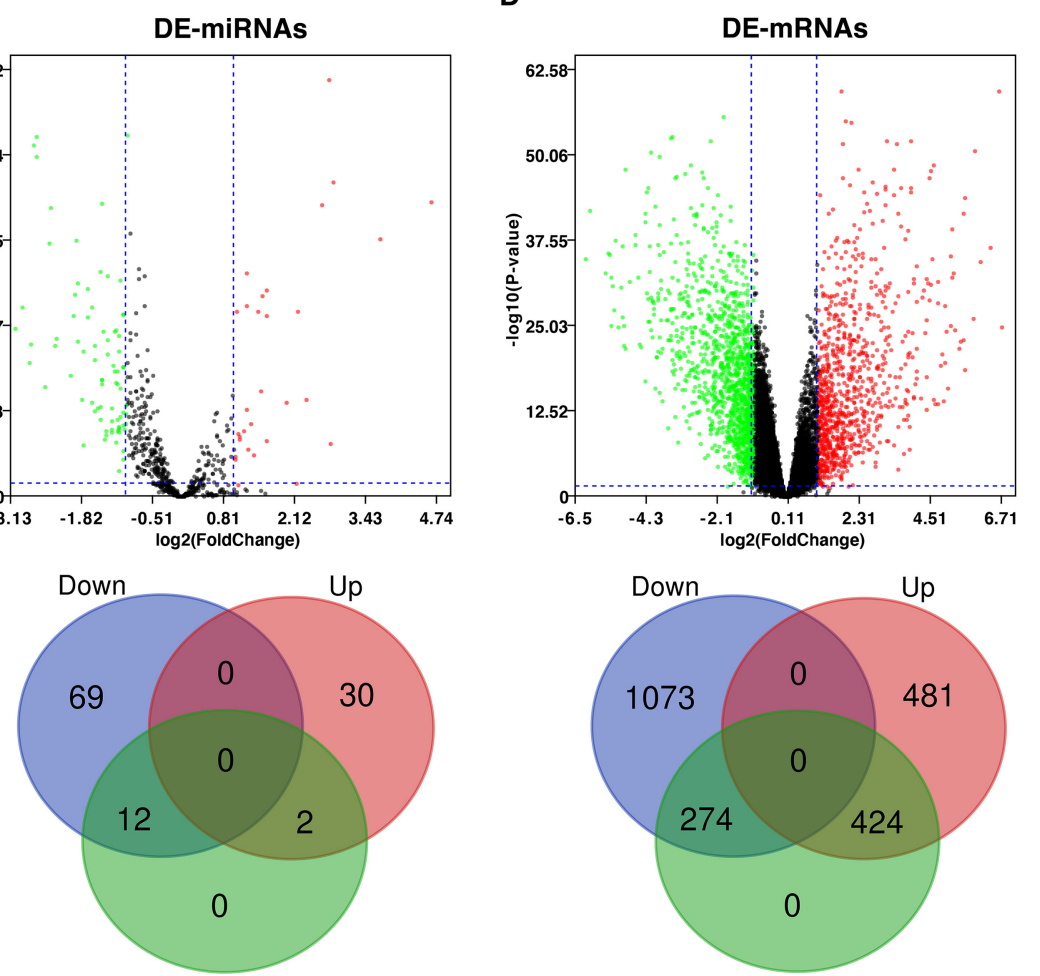

ce targets

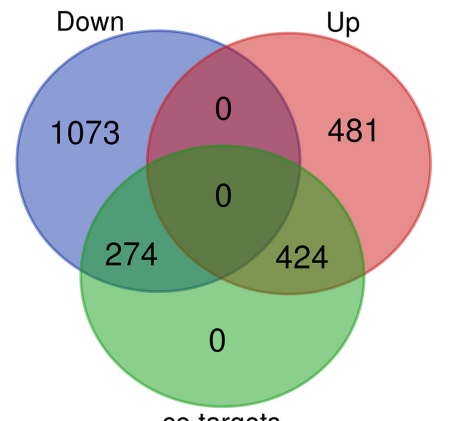

ce targets

FIGURE 1 | Differential expression analysis and prediction of potential circRNA-associated competing endogenous RNA (ceRNA) regulatory relationships. (A) The workflow of constructing a circRNA-associated ceRNA network and the establishment of novel prognostic DE-mRNA signature and nomogram to predict risk of 
FIGURE 1 | progression in post-surgical papillary thyroid carcinoma (PTC). (B) The volcano plot and the Venn diagram show that 138 differentially expressed circRNAs (DE-circRNAs) are identified in PTC (117 upregulated and 21 downregulated). Of these, 54 DE-circRNAs are associated with potential ceRNA regulatory relationships. (C) The volcano plot and the Venn diagram show that 113 differentially expressed miRNAs (DE-miRNAs) are identified in PTC (32 upregulated and 81 downregulated). Of these, 14 DE-miRNAs are associated with potential ceRNA regulatory relationships. (D) The volcano plot and the Venn diagram show that 2,252 differentially expressed mRNAs (DE-mRNAs) were identified in PTC (905 upregulated and 1,347 downregulated). Of these, 968 DE-mRNAs are associated with potential ceRNA regulatory relationships.

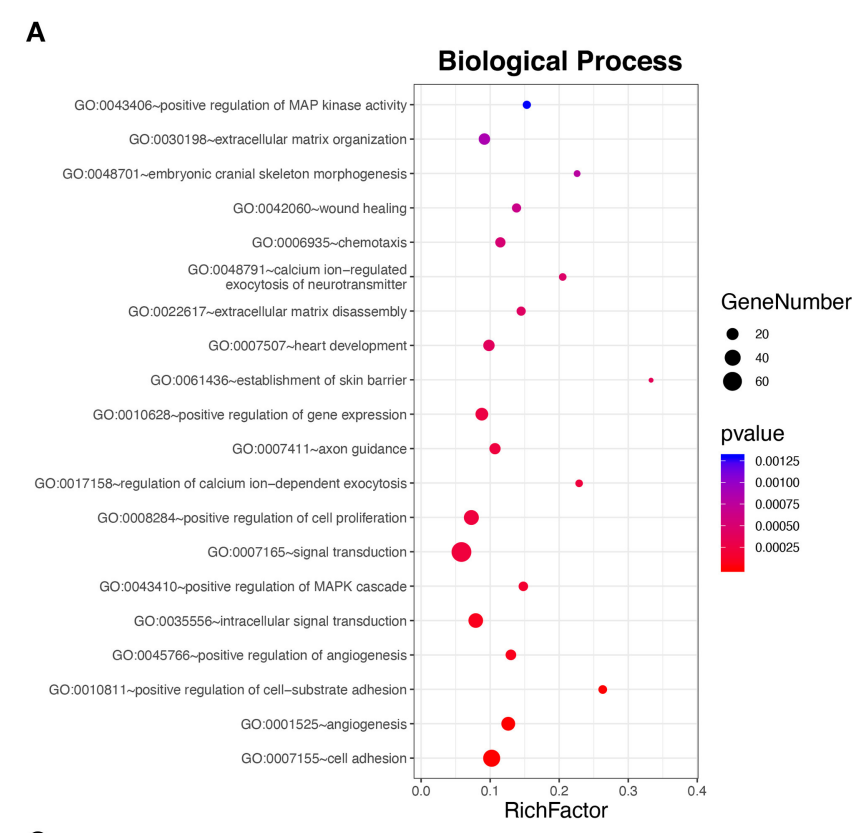

C

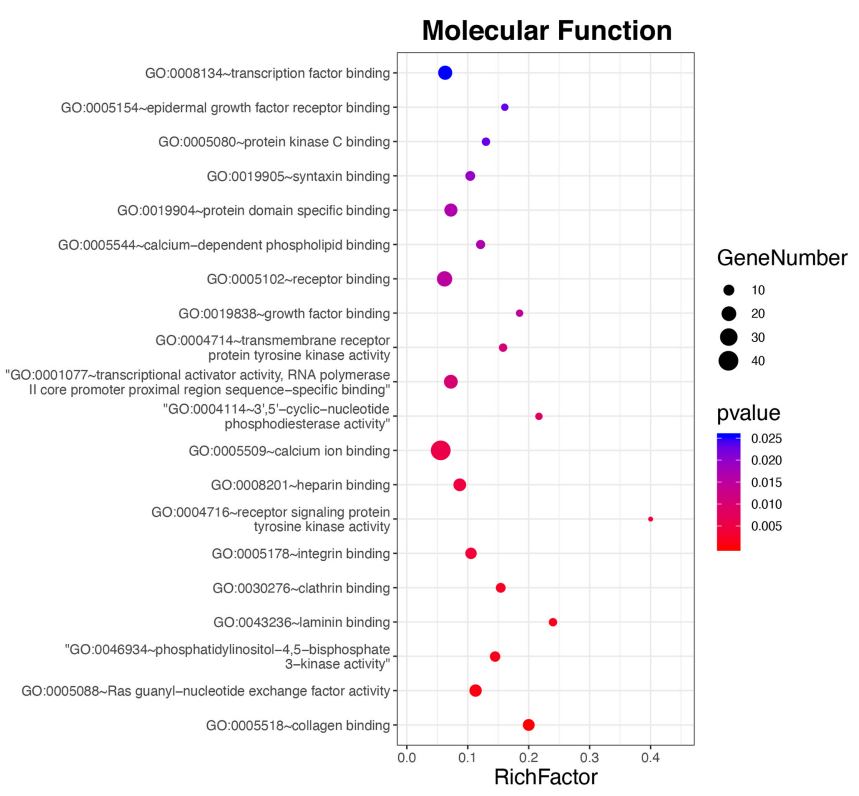

B

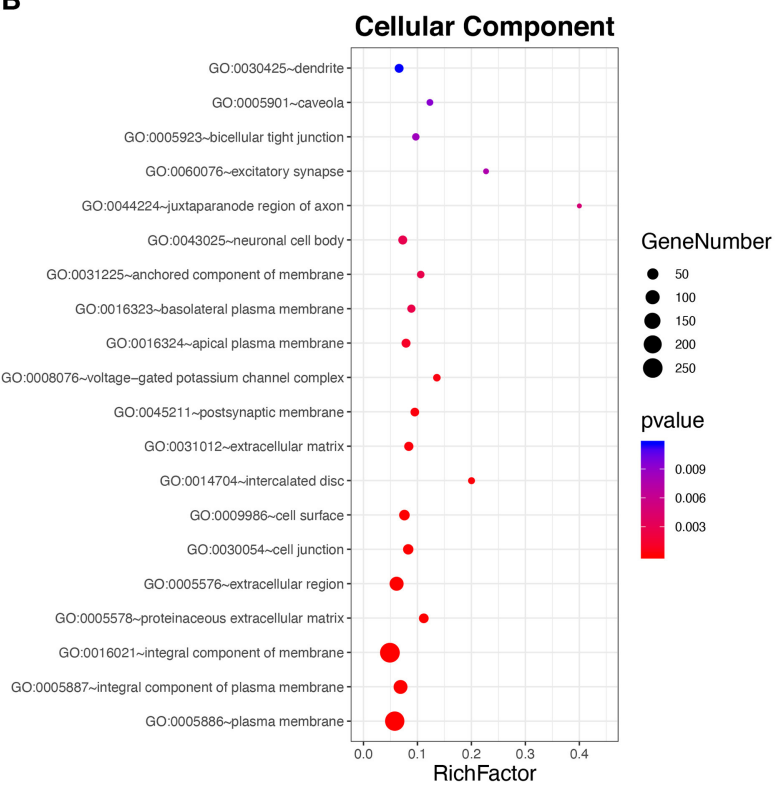

D

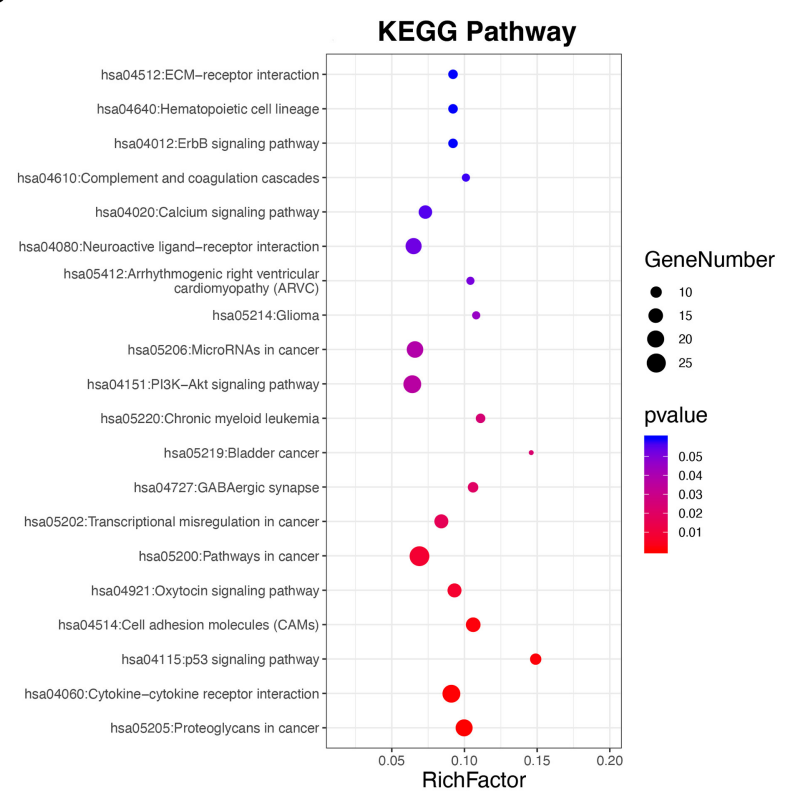

FIGURE 2 | Functional enrichment analysis of the 698 DE-mRNAs with potential circRNA-associated ceRNA regulatory relationships. (A) Top 20 most enriched biological processes of the DE-mRNAs. (B) Top 20 most enriched cellular components of the DE-mRNAs. (C) Top 20 most enriched molecular functions of the DE-mRNAs. (D) Top 20 most enriched pathways of the DE-mRNAs. 


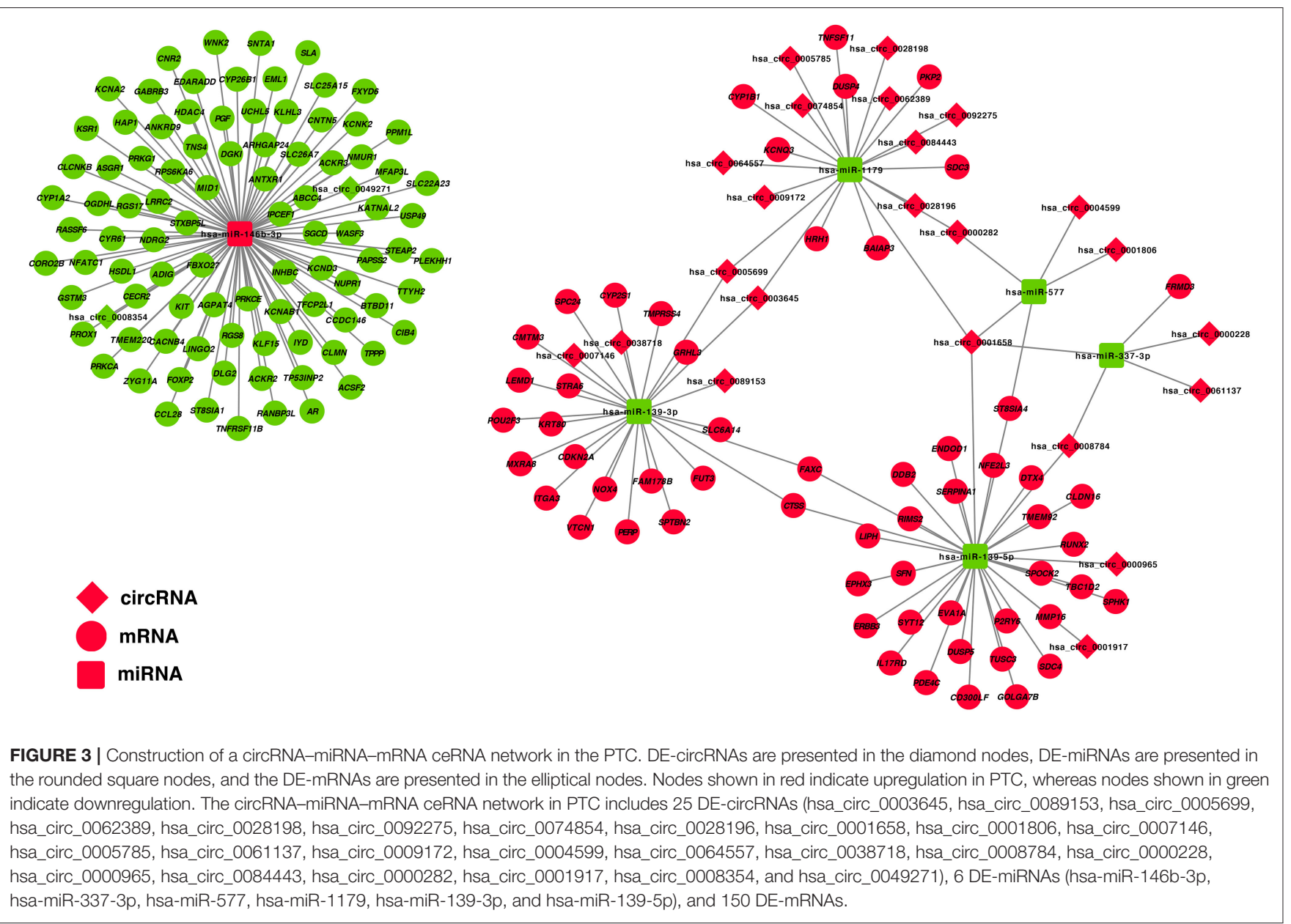

\section{Identification of Hub PFI-Related DE-mRNAs Regulated by the circRNA-Associated ceRNA Mechanism and Establishment of a Prognostic DE-mRNA Signature}

To identify hub PFI-related DE-mRNAs within the ceRNA subnetwork, 492 TCGA cases with follow-up $>30$ days were randomly divided into the training dataset and the validation dataset in a 7:3 ratio (Table 2). The training dataset was employed to identify hub DE-mRNAs related to PFI using LASSO-Cox regression. Subsequently, six DE-mRNAs, including CLCNKB, FXBO27, FXYD6, RIMS2, SPC24, and CDKN2A, were identified as hub PFI-related DE-mRNAs (Figures 5A,B). ceRNA regulatory relationships of the six hub DE-mRNAs are shown in Figure 5C. Downregulated CLCNKB, FBXO27, and FXYD6 with $\mathrm{HR}<1$ were negatively regulated by hsamiR-146b-3p. Upregulated RIMS2 with HR >1 was negatively regulated by hsa-miR-139-5p. Moreover, upregulated SPC24 and $C D K N 2 A$ with $\mathrm{HR}>1$ were negatively regulated by hsa-miR-139-3p. Kaplan-Meier curves for the six hub PFIrelated DE-mRNAs based on the optimal cutoff values in PTC are shown in Figures 5D-I. The differential expression of the hub DE-mRNAs between PTC and normal thyroid tissues was validated using GEPIA (Supplementary Figure 1A), and differential expression of corresponding DE-circRNAs and DE-miRNAs is shown in Supplementary Figures 1B-E. A DE-mRNA signature based on the LASSO-Cox regression model coefficients $(\beta)$ multiplied with corresponding normalized mRNA expression level was established. The risk score was equal to $-[(0.09231) *$ expression value of $C L C N K B]-[(0.14778) *$ expression value of FBXO27] - [(0.12888) * expression value of FXYD6 $]+[(0.11113) *$ expression value of RIMS2] $+[(0.56913)$ * expression value of SPC24] $+[(0.05512) *$ expression value of CDKN2A]. The risk score of each case was calculated with this formula. The C-index of the DE-mRNA signature in predicting PFI of PTC was 0.741 (95\% CI, 0.564-0.918). ROC curves revealed that the AUCs of the DE-mRNA signature in predicting 1-, 2-, 3-, 4-, and 5-year PFI were 0.793 (95\% CI, 0.652-0.934), 0.769 (95\% CI, 0.676-0.863), 0.701 (95\% CI, $0.588-0.814), 0.687$ (95\% CI, 0.543-0.831), and 0.712 (95\% CI, $0.579-0.845$ ), respectively (Figure 6A). Thereafter, patients were divided into a high-risk and a low-risk group according to the optimal cutoff value determined by X-tile. Kaplan-Meier analysis revealed that high-risk patients with significantly poorer prognosis could be discriminated by the DE-mRNA signature 


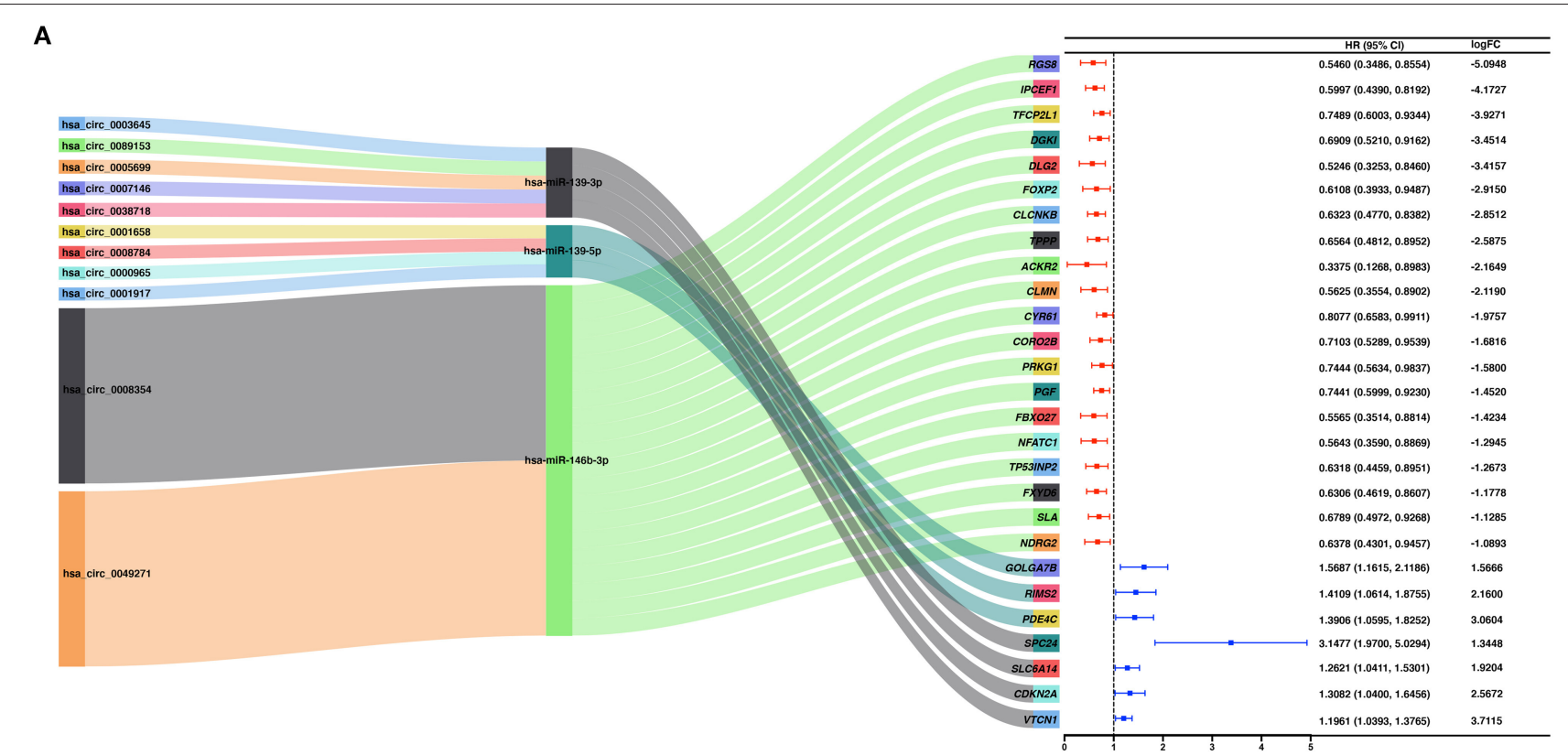

B

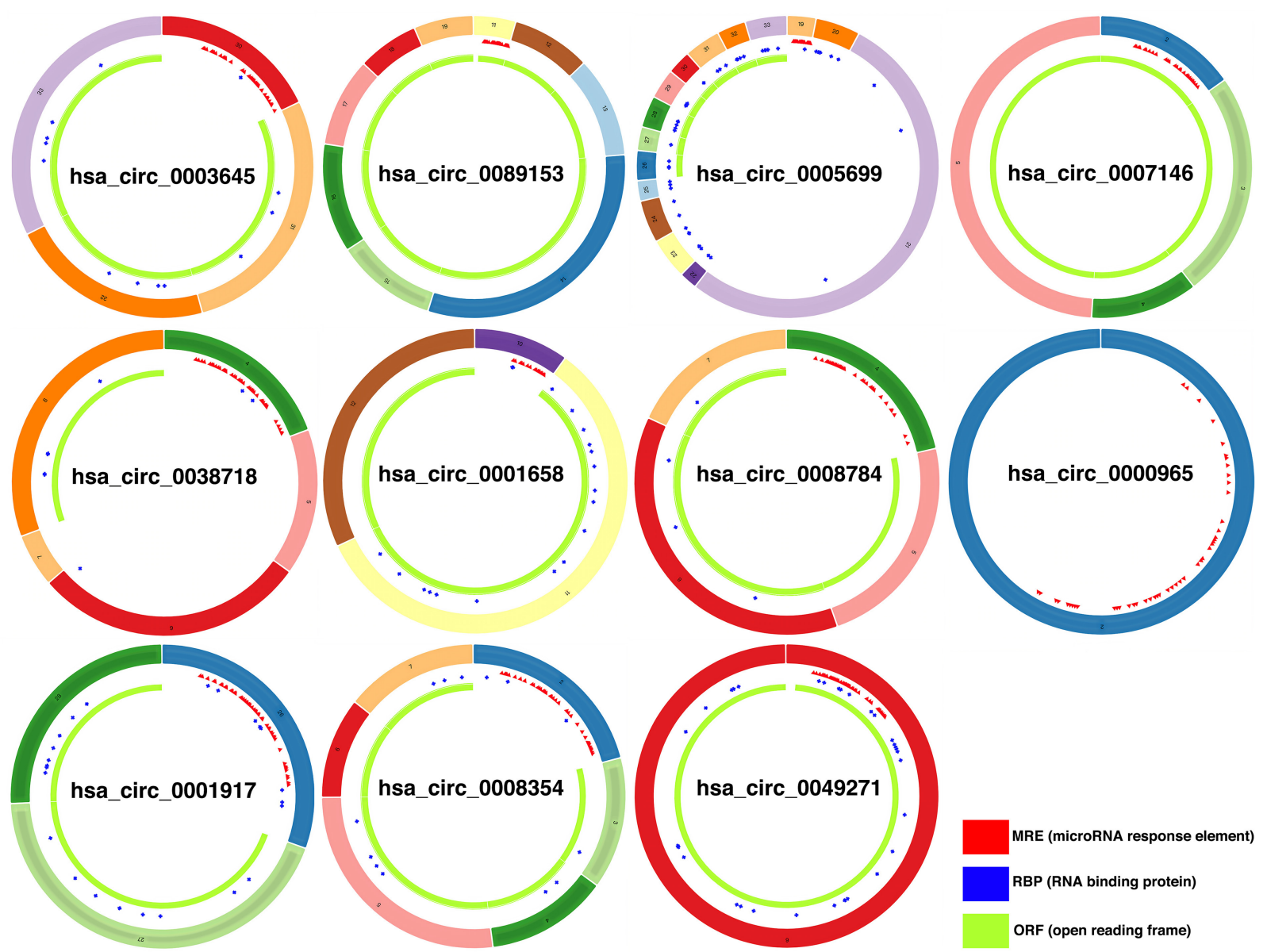

FIGURE 4 | Construction of a ceRNA regulatory subnetwork associated with progression-free interval (PFI) in PTC. (A) The Sankey diagram presents the regulatory relationship within the ceRNA regulatory subnetwork associated with PFI in PTC (left). Forest plot of hazard ratio (HR) presenting the prognostic value of the 
FIGURE 4 | PFI-related DE-mRNAs within the ceRNA subnetwork and the corresponding logFC values for differential expression (PTC vs. normal) (right). (B) Basic structures of the 11 DE-circRNAs within the ceRNA subnetwork. The structural patterns of hsa_circ_0003645, hsa_circ_0089153, hsa_circ_0005699,

hsa_circ_0007146, hsa_circ_0038718, hsa_circ_0001658, hsa_circ_0008784, hsa_circ_0000965, hsa_circ_0001917, hsa_circ_0008354, and hsa_circ_0049271 provided by Cancer-Specific CircRNA Database (CSCD) are shown. The microRNA response element (MRE) is presented in red. The RNA-binding protein (RBP) is presented in blue and the open reading frame (ORF) is presented in green.

TABLE 1 | Basic characteristics of the 11 differently expressed circRNAs.

\begin{tabular}{|c|c|c|c|c|c|c|}
\hline CircRNA ID & $\log \mathrm{FC}$ & Regulation & Position & Strand & Spliced length & Parental gene symbol \\
\hline hsa_circ_0003645 & 2.46 & Up & chr16:19656207-19663412 & + & 356 & C16orf62 \\
\hline hsa_circ_0089153 & 2.37 & Up & chr9:134011326-134022971 & + & 1,102 & NUP214 \\
\hline hsa_circ_0005699 & 2.32 & Up & chr16:19627435-19663412 & + & 1,198 & C16orf62 \\
\hline hsa_circ_0001658 & 1.57 & Up & chr6:157357968-157406039 & + & 4,8071 & $A R I D 1 B$ \\
\hline hsa_circ_0007146 & 1.48 & Up & chr16:5077135-5078186 & - & 420 & NAGPA \\
\hline hsa_circ_0000965 & 1.17 & Up & chr19:58472758-58476205 & - & 3,447 & C19orf18 \\
\hline hsa_circ_0001917 & 1.02 & Up & chrX:41519691-41530783 & - & 402 & CASK \\
\hline hsa_circ_0008354 & -1.03 & Down & chr19:34981280-34986676 & + & 485 & WTIP \\
\hline hsa_circ_0049271 & -1.34 & Down & chr19:10610070-10610756 & - & 686 & KEAP1 \\
\hline
\end{tabular}

(Figure 6D). As the incremental increase of risk score and the deterioration of prognosis occurred, the expression of FXYD6, $C L C N K B$, and $F B X O 27$ gradually decreased, and the expression of RIMS2, SPC24, and CDKN2A gradually increased (Figure 6G). In general, the prognostic DE-mRNA signature based on hub PFI-related DE-mRNAs within the ceRNA subnetwork was accurate in predicting PFI of PTC in the training dataset.

\section{Validation of the DE-mRNA Signature in Predicting PFI of PTC}

The performance of the DE-mRNA signature in predicting PFI of the PTC was validated in the validation dataset and the entire TCGA-THCA dataset. The C-index of the DE-mRNA signature in predicting PFI of PTC was 0.665 (95\% CI, 0.3910.939 ) and 0.716 (95\% CI, 0.565-0.867), respectively. ROC curves revealed that in the validation dataset, AUCs of the DEmRNA signature in predicting 1-, 2-, 3-, 4-, and 5-year PFI were 0.661 (95\% CI, 0.433-0.890), 0.647 (95\% CI, 0.512-0.783), 0.684 (95\% CI, 0.538-0.830), 0.710 (95\% CI, 0.554-0.866), and 0.657 (95\% CI, 0.476-0.838), respectively (Figure 6B). Moreover, in the entire TCGA-THCA dataset, AUCs of the DE-mRNA signature in predicting 1-, 2-, 3-, 4-, and 5-year PFI were 0.744 (95\% CI, 0.617-0.870), 0.728 (95\% CI, 0.649-0.806), 0.695 (95\% CI, 0.606-0.784), 0.696 (95\% CI, 0.589-0.804), and 0.696 (95\% CI, 0.591-0.801), respectively (Figure 6C). Thereafter, patients in each dataset were divided into a high-risk and a low-risk group according to the optimal cutoff values determined by X-tile. Kaplan-Meier analysis revealed that high-risk patients with significantly poorer prognosis could be discriminated by the DE-mRNA signature in both datasets (Figures 6E,F). Moreover, heatmaps revealed that the expression of the six DE-mRNAs gradually changed along with risk score increase and prognosis deterioration (Figures 6H,I). The prognostic DEmRNA signature based on the hub PFI-related DE-mRNAs within the ceRNA subnetwork was confirmed to be robust in predicting PFI of PTC in the validation dataset and the entire TCGA-THCA dataset.

\section{Analysis of Molecular, Clinical, and Mutational Relevance of the DE-mRNA Signature}

To explore the underlying molecular mechanism of the DEmRNA signature, the entire TCGA-THCA dataset was divided into a high-risk and a low-risk group based the optimal cutoff value of the DE-mRNA signature determined by X-tile. GSEAs were utilized to compare molecular alteration in the high-risk group and the low-risk group against previously annotated gene sets in the Molecular Signatures Database v7.1. Regarding KEGG pathways, molecular alteration in the high-risk group was significantly enriched in the p53 signaling pathway, cell cycle, etc. (Figures 7A,B). Analysis of hallmark gene sets further revealed that molecular alteration in the high-risk group was significantly associated with genes encoding cell cycle-related targets of E2F transcription factors (Figure 7C). HALLMARK_MYC_TARGETS_V1 and HALLMARK_MYC_TARGETS_V2 was also significantly enriched indicating that the molecular alteration in the high-risk group was potentially regulated by MYC (Figures 7D,E). Full results of the GSEA are shown in Supplementary Table 8.

Analysis of clinical relevance revealed that the risk score increased significantly along with the escalation of T stage $(P<$ 0.05 ; Figure 7F). Patients with lymph node and distal metastasis had a significantly higher risk score $(P<0.05$; Figures $7 \mathbf{G}, \mathbf{H})$. Regarding AJCC stage, advanced stages (III and IV) were 
TABLE 2 | Clinical features of patients in TCGA-THCA dataset.

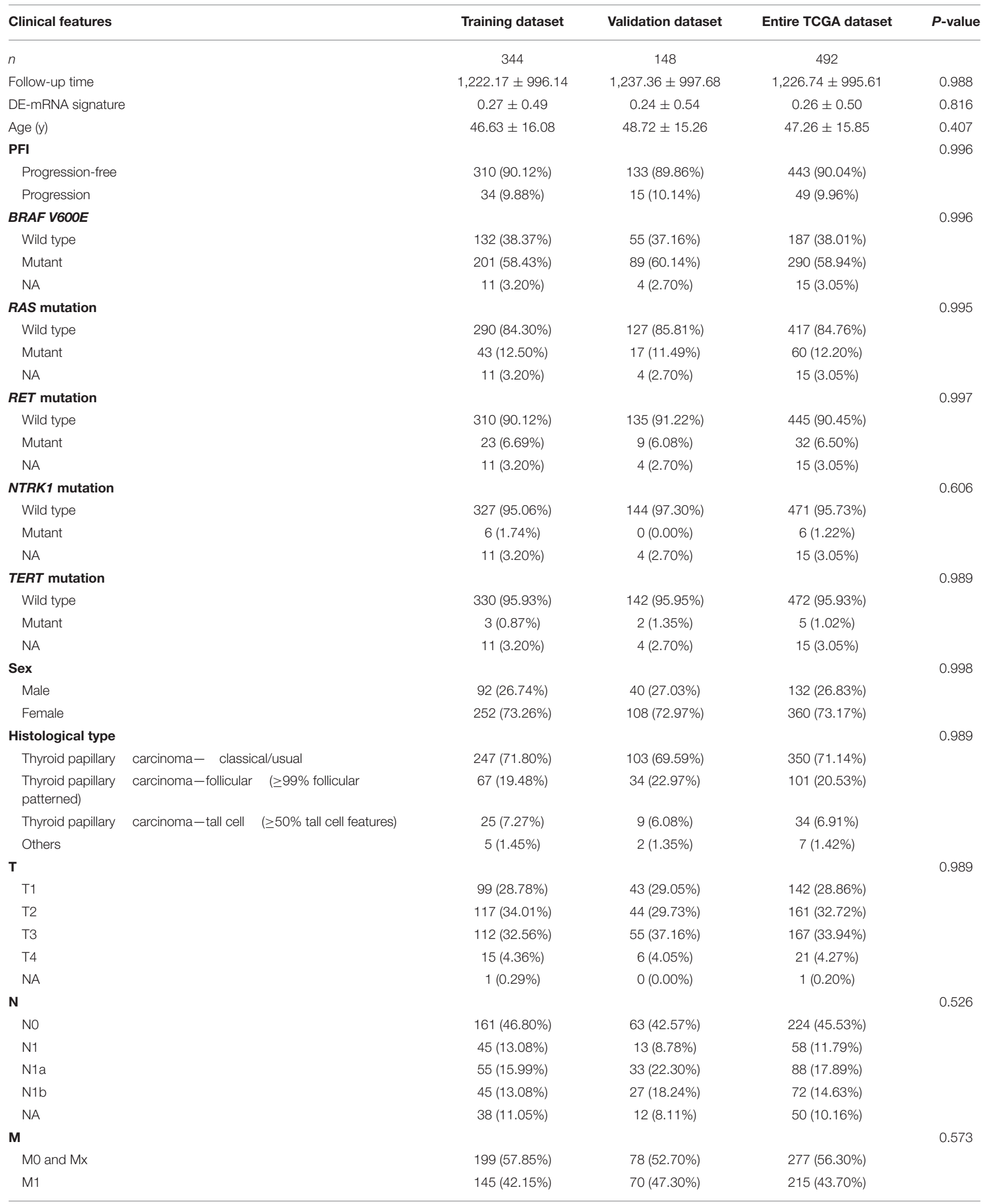


TABLE 2 | Continued

\begin{tabular}{|c|c|c|c|c|}
\hline Clinical features & Training dataset & Validation dataset & Entire TCGA dataset & $P$-value \\
\hline AJCC stage & & & & 0.519 \\
\hline Stage I & 207 (60.17\%) & $72(48.65 \%)$ & $279(56.71 \%)$ & \\
\hline Stage II & 35 (10.17\%) & 15 (10.14\%) & 50 (10.16\%) & \\
\hline Stage III & 66 (19.19\%) & 42 (28.38\%) & 108 (21.95\%) & \\
\hline Stage IV & 35 (10.17\%) & $18(12.16 \%)$ & 53 (10.77\%) & \\
\hline NA & $1(0.29 \%)$ & $1(0.68 \%)$ & $2(0.41 \%)$ & \\
\hline Residual tumor & & & & 0.922 \\
\hline Ro & $258(75.00 \%)$ & 117 (79.05\%) & 375 (76.22\%) & \\
\hline $\mathrm{Rx}$ & $48(13.95 \%)$ & $14(9.46 \%)$ & $62(12.60 \%)$ & \\
\hline $\mathrm{R} 1$ & 35 (10.17\%) & $16(10.81 \%)$ & $51(10.37 \%)$ & \\
\hline $\mathrm{R} 2$ & $3(0.87 \%)$ & $1(0.68 \%)$ & $4(0.81 \%)$ & \\
\hline Extrathyroid extension & & & & 0.997 \\
\hline None & $230(66.86 \%)$ & 95 (64.19\%) & $325(66.06 \%)$ & \\
\hline Minimal (T3) & $90(26.16 \%)$ & $42(28.38 \%)$ & $132(26.83 \%)$ & \\
\hline Moderate or advanced (T4) & $11(3.20 \%)$ & $6(4.05 \%)$ & 17 (3.46\%) & \\
\hline NA & 13 (3.78\%) & 5 (3.38\%) & 18 (3.66\%) & \\
\hline Multifocality & & & & 0.994 \\
\hline Unifocal & $178(51.74 \%)$ & $80(54.05 \%)$ & $258(52.44 \%)$ & \\
\hline Multifocal & 159 (46.22\%) & 65 (43.92\%) & 224 (45.53\%) & \\
\hline NA & 7 (2.03\%) & $3(2.03 \%)$ & $10(2.03 \%)$ & \\
\hline Anatomic site & & & & 0.845 \\
\hline Unilateral & 267 (77.62\%) & 113 (76.35\%) & 380 (77.24\%) & \\
\hline Isthmus & $14(4.07 \%)$ & $8(5.41 \%)$ & $22(4.47 \%)$ & \\
\hline Bilateral & $61(17.73 \%)$ & 24 (16.22\%) & 85 (17.28\%) & \\
\hline NA & $2(0.58 \%)$ & $3(2.03 \%)$ & $5(1.02 \%)$ & \\
\hline
\end{tabular}

associated with a higher risk score than early stages (I and II) $(P$ $<0.05$; Figure 7I). Patients with microscopic and macroscopic residual tumors also had a significantly higher risk score, further indicating the relevance of the DE-mRNA signature to the aggressiveness of PTC $(P<0.05$; Figure $7 \mathrm{~J})$. Analysis of histological subtypes revealed that aggressive subtypes of PTC, including tall cell, hobnail variant, and columnar cell carcinoma, were associated with a significantly higher risk score $(P<0.05$; Figure 7K).

Analysis of mutational relevance revealed that the $B R A F$ V600E mutation and RAS mutation was associated with a significantly higher and lower risk score, respectively $(P<0.05$; Figures 7L,M). The mutational pattern of PTC further revealed that CLCNKB, FBXO27, and FXYD6 had a relatively lower expression level in samples with the BRAF V600E mutation and were negatively associated with hsa-miR-146b-3p expression (Figure 7N). Alternatively, SPC24 and CDKN2A negatively regulated by hsa-miR-139-3p had a relatively higher expression level in samples with the BRAF V600E mutation. RIMS2 also had a relatively higher expression level in samples with the $B R A F$ V600E mutation and was negatively associated with hsa-miR139-5p expression. Regarding the RAS mutation, the six DEmRNAs and the corresponding miRNA regulators had opposite expression patterns to the BRAF V600E mutation.

\section{Validation of the Independent Prognostic Role of the DE-mRNA Signature in PTC}

A total of 389 cases from the entire TCGA-THCA dataset with complete clinical information were utilized to identify prognostic factors associated with PFI in PTC. Clinical information for analysis included DE-mRNA signature, age, PFI status, mutational status of BRAF V600E, RAS, RET, NTRK1, and TERT, sex, histological subtype, $\mathrm{T}$ stage, $\mathrm{N}$ stage, $\mathrm{M}$ stage, AJCC stage, residual tumor status, extrathyroidal extension, multifocality, and anatomic site. Reasons for exclusion are listed in Supplementary Table 9. Baseline characteristics of patients who were included for prognostic factor evaluation are shown in Table 3. Patients in the high-risk group had significantly worse prognosis; higher T stage, N stage, $\mathrm{M}$ stage, and AJCC stage; and a larger portion with the BRAF V600E mutation and extrathyroidal extension. Tumors of the high-risk group were also more likely to be unifocal. Univariate Cox regression analysis revealed that DE-mRNA signature, age, histological type, aggressive subtype, $\mathrm{T}$ stage, N stage, AJCC stage, and neoplasm largest dimension were prognostic factors associated with PFI of PTC $(P<0.05$; Table 4). Adjustment for parameters with $P<0.25$ based on univariate analysis and multivariate Cox regression analysis further validated that the DE-mRNA signature was an independent prognostic factor associated with PFI in PTC (Table 5). The 
A

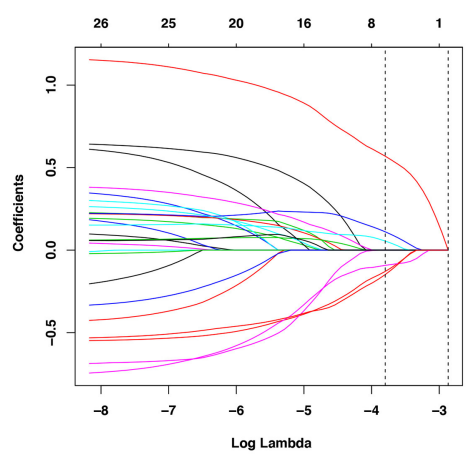

D

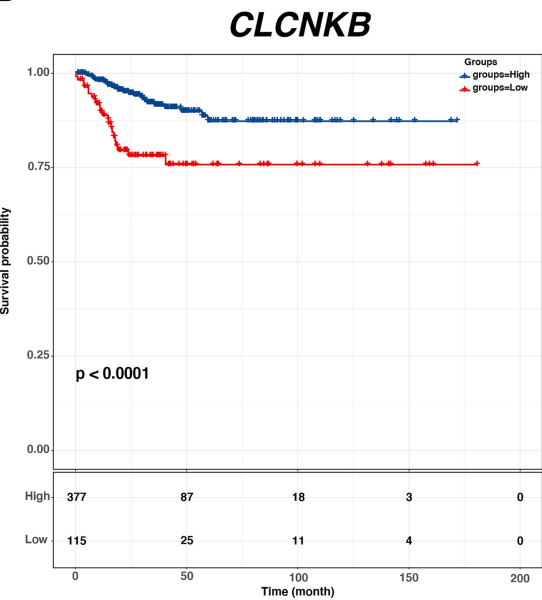

G

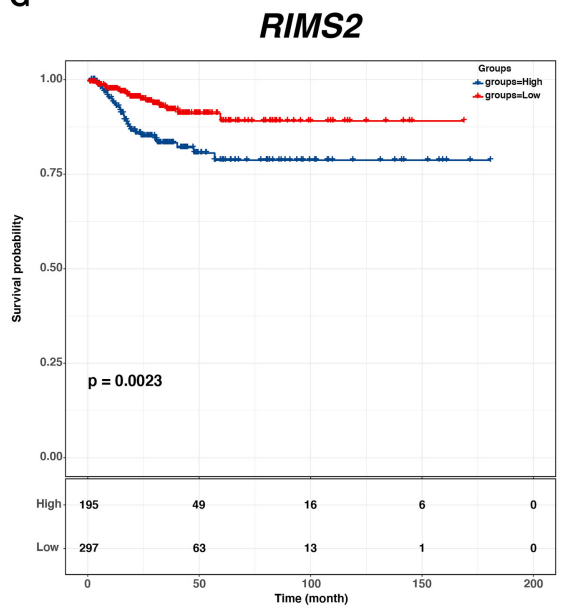

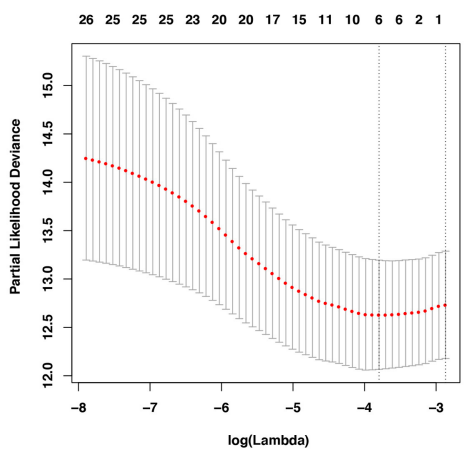

E
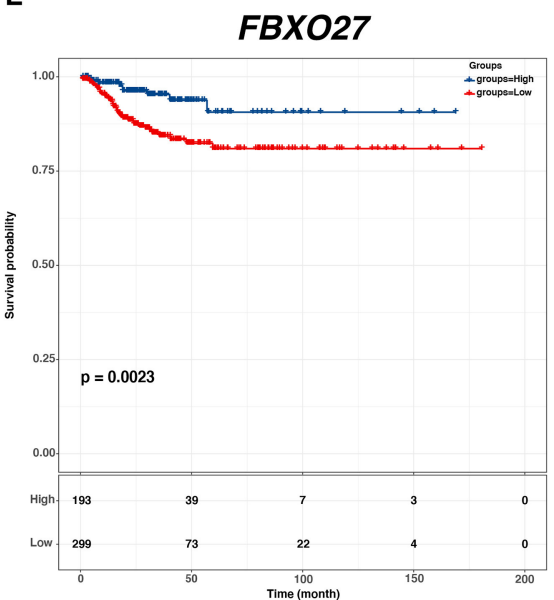

H
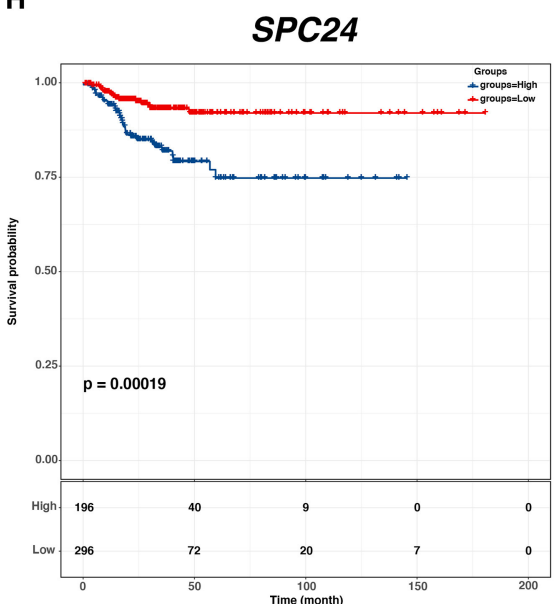

C

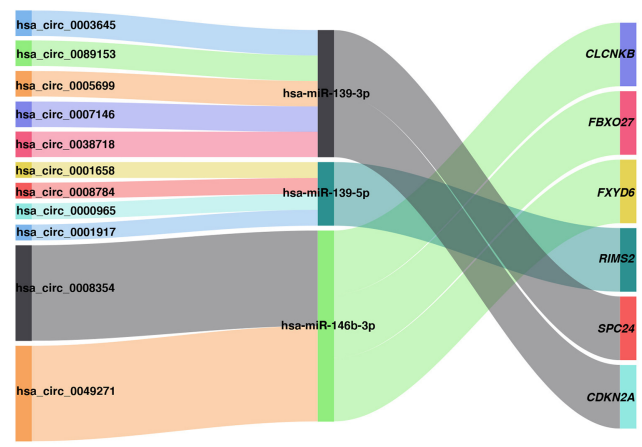

F

FXYD6

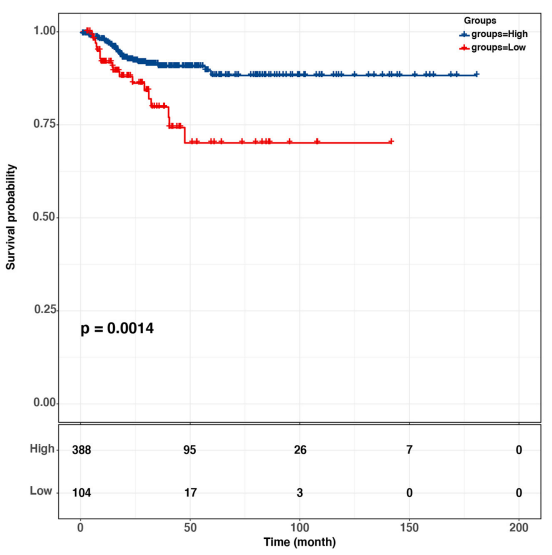

I

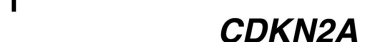

FIGURE 5 | Identification of hub PFI-related DE-mRNAs regulated by ceRNA mechanism and establishment of DE-mRNA signature. (A) LASSO coefficient profiles of the 27 prognostic DE-mRNAs within the subnetwork. (B) LASSO deviance profiles of the 27 prognostic DE-mRNAs within the subnetwork. (C) The Sankey diagram presents the regulatory relationship between the hub PFI-related DE-mRNAs and the corresponding DE-miRNAs and DE-circRNAs. (D-I) Kaplan-Meier curves for the hub DE-mRNAs related to PFI based on the optimal cutoff values in PTC. The horizontal axis shows the follow-up time in months, and the vertical axis shows the probability of progression-free survival. 


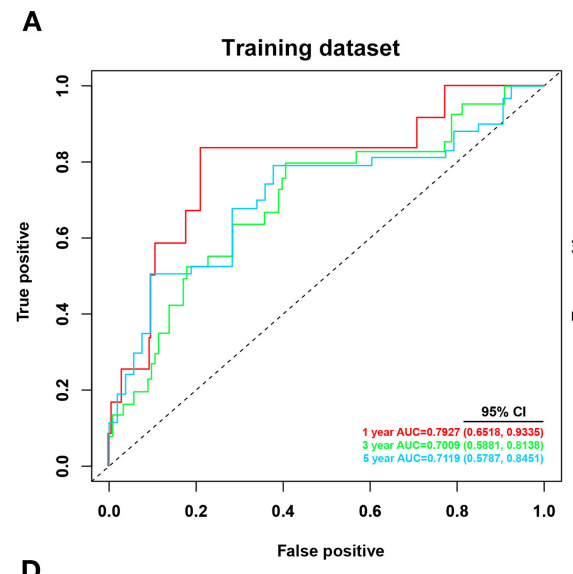

D

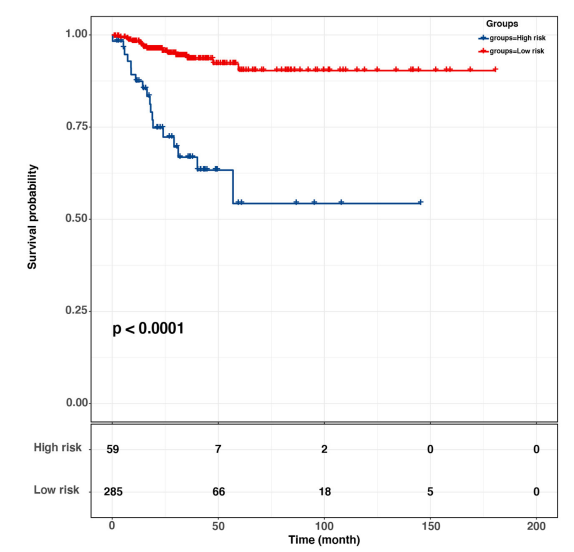

G
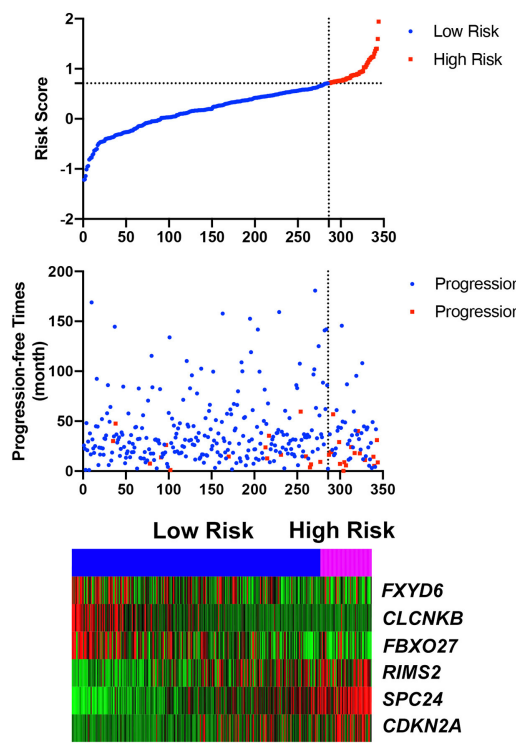

B

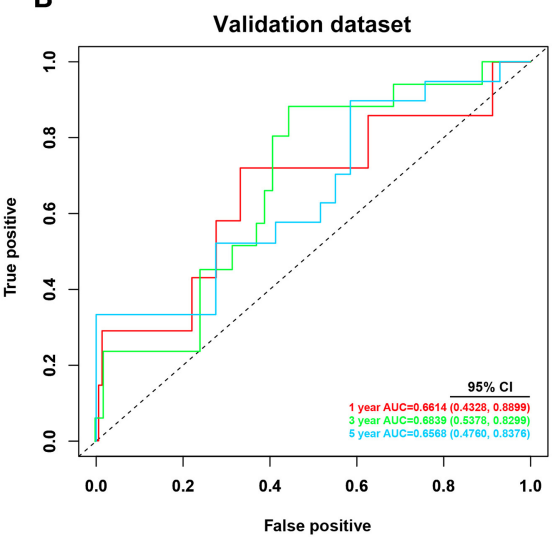

E

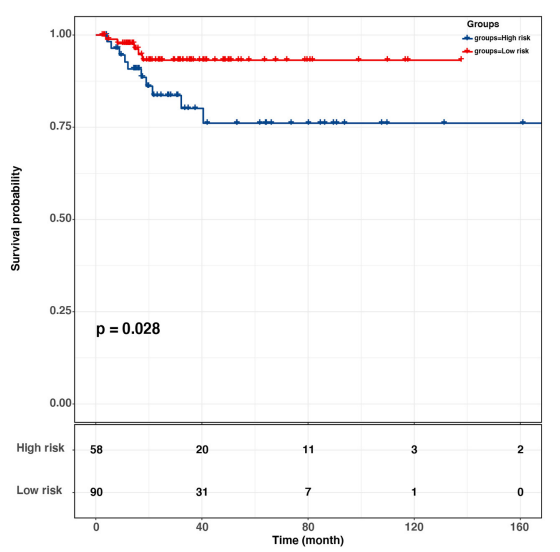

H
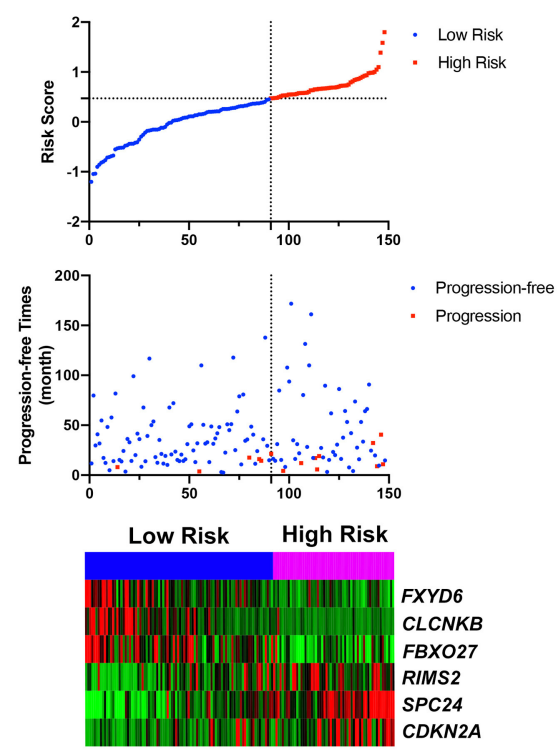

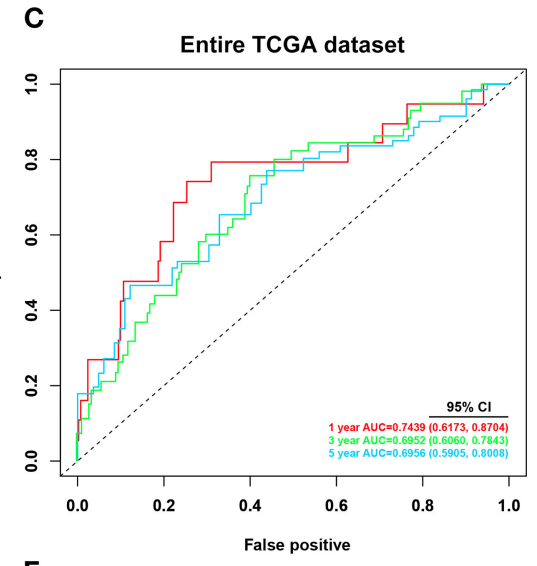

$\mathbf{F}$

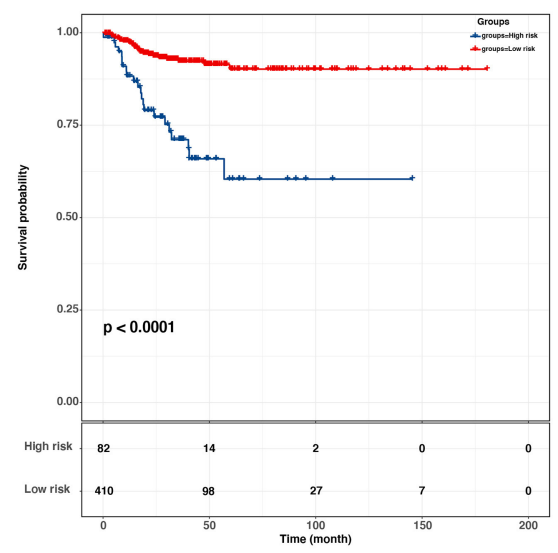

I
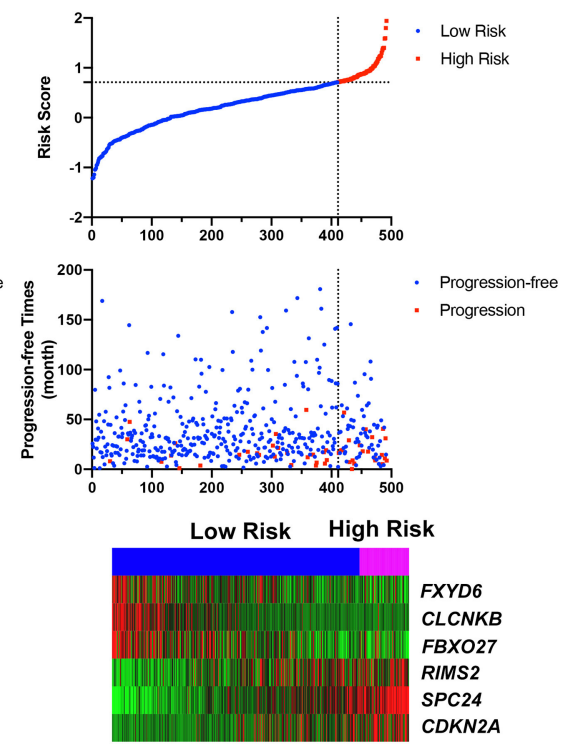

FIGURE 6 | Validation of the predicting performance of the DE-mRNA signature. (A-C) The time-dependent receiver operating characteristic (ROC) curves for 1-, 3-, and 5 -year PFI predicted by the DE-mRNA signature in the training dataset, the validation dataset, and the entire TCGA-THCA dataset, respectively. (D-F) The Kaplan-Meier curves for the DE-mRNA signature based on the optimal cutoff value in the training dataset, the validation dataset, and the entire TCGA-THCA dataset, respectively. The horizontal axis shows the follow-up time in months, and the vertical axis shows the probability of progression-free survival. (G-I) The trends in expression profiles of the six hub DE-mRNAs (bottom) and the deterioration of prognosis (middle), along with the incremental risk of progression based on the DE-mRNA signature (upper) in the training dataset, the validation dataset, and the entire TCGA-THCA dataset, respectively. 


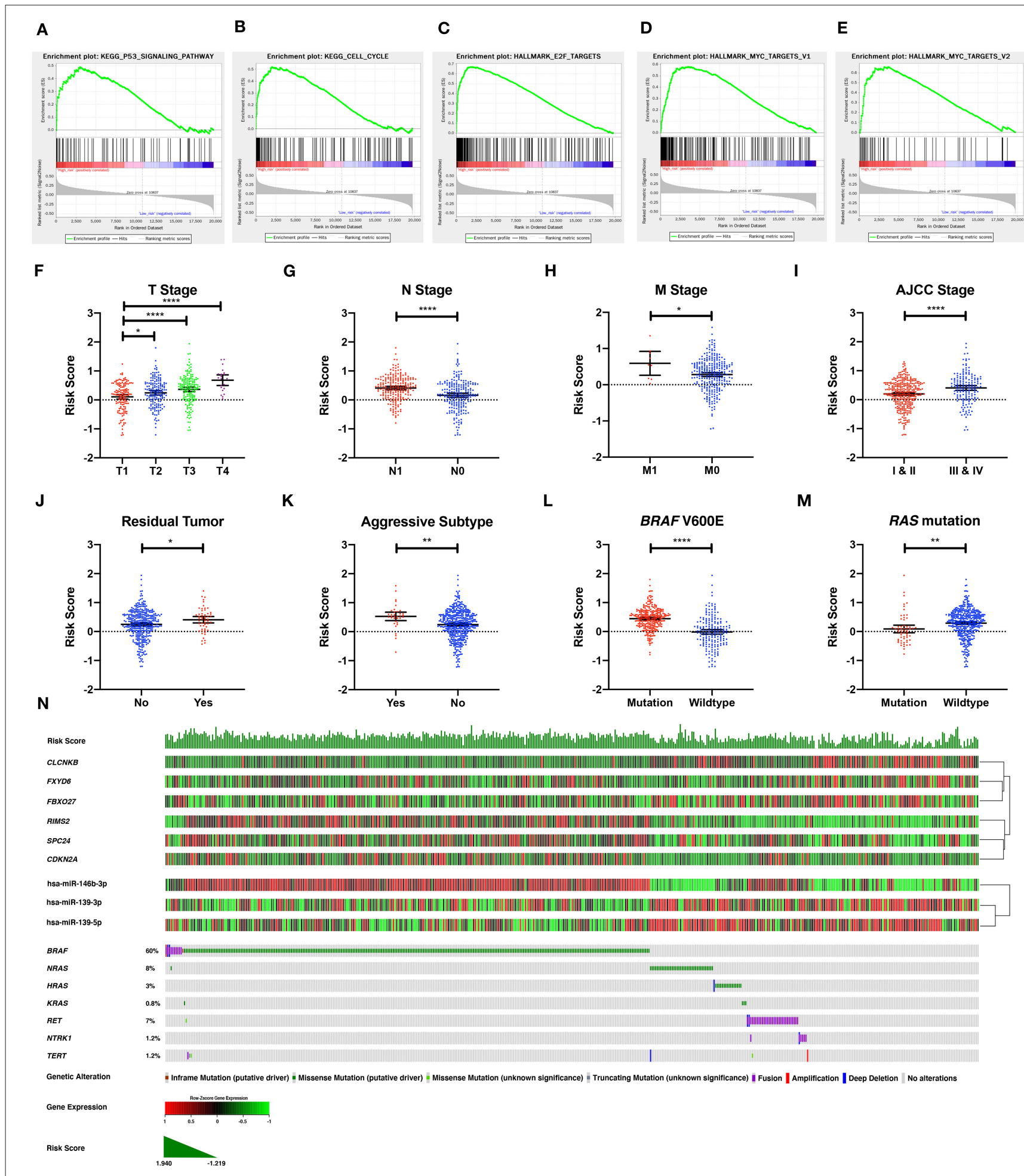

FIGURE 7 | The molecular, clinical, and mutational relevance of the DE-mRNA signature. (A-E) Top functional gene sets enriched in the high-risk group predicted by the DE-mRNA signature based on the optimal cutoff values in PTC. (F) The distribution of the risk score calculated by the DE-mRNA signature in different groups of $T$ stages in the TCGA-THCA dataset. (G) The distribution of the risk score calculated by the DE-mRNA signature in different groups of N stages in the TCGA-THCA dataset. (H) The distribution of the risk score calculated by the DE-mRNA signature in different groups of M stages in the TCGA-THCA dataset. (I) The distribution of the risk score calculated by the DE-mRNA signature in different groups of AJCC stages in the TCGA-THCA dataset. (J) The distribution of the risk score calculated by the DE-mRNA signature in different groups of residual tumor statuses in the TCGA-THCA dataset. (K) The distribution of the risk score calculated by the DE-mRNA 
FIGURE 7 | signature in groups of aggressive and non-aggressive subtypes in the TCGA-THCA dataset. (L) The distribution of the risk score calculated by the DE-mRNA signature in different groups of BRAF V600E mutation status in the TCGA-THCA dataset. (M) The distribution of the risk score calculated by the DE-mRNA signature in different groups of RAS mutation status in the TCGA-THCA dataset. (N) The relationship among the DE-mRNA signature, the expression profiles of the six hub DE-mRNAs (CLCNKB, FXYD6, FBXO27, RIMS2, SPC24, and CDKN2A) and corresponding DE-miRNAs (hsa-miR-146b-3p, hsa-miR-139-3p, and hsa-miR-139-5p) and the mutational profiles (BRAF, RAS, RET, NTRK1, and TERT) of PTC. Data of genetic alteration were obtained from the cBioPortal for Cancer Genomics (https://www.cbioportal.org). ${ }^{\star} P<0.05,{ }^{\star \star} P<0.01$, and ${ }^{\star \star \star \star} P<0.0001$.

RAS mutation was also identified to be an independent prognostic factor.

\section{Building and Validation of a DE-mRNA Signature-Based Prognostic Nomogram}

Based on cases from the entire TCGA-THCA dataset with complete clinical information, a prognostic nomogram was established with the DE-mRNA signature and prognostic clinical factors using stepwise Cox regression (Figure 8A). Factors involved in the nomogram included the DE-mRNA signature, $R A S$ mutation status, aggressive subtype, $\mathrm{N}$ stage, and tumor size. The C-index of the nomogram in predicting PFI of PTC was 0.790 (95\% CI, 0.652-0.927). The calibration curve revealed that the predicted risk of progression by the nomogram was close to the observed risk of progression (Figure 8C). Patients were subsequently divided into the high-risk and the low-risk group according to the optimal cutoff value determined by $\mathrm{X}$ tile. Kaplan-Meier analysis revealed that high-risk patients had significantly poorer prognosis than low-risk patients (Figure 8B). The ATA risk stratification system is recommended to estimate the risk of post-surgical recurrent disease in PTC. MACIS score and AJCC staging system are employed to predict post-surgical mortality of PTC. The accuracy of nomogram in predicting PFI of PTC was analyzed with ROC curves using the ATA risk stratification system, MACIS score, and AJCC staging system as reference (Figures $\mathbf{8 D}-\mathbf{H}$ ). The AUCs of the nomogram in predicting 1-, 2-, 3-, 4-, and 5-year PFI were 0.855 (95\% CI, $0.779-0.932$ ), 0.779 (95\% CI, 0.699-0.860), 0.799 (95\% CI, 0.7220.877 ), 0.836 (95\% CI, 0.762-0.909), and 0.812 (95\% CI, 0.7180.907), respectively. The AUCs of the ATA risk stratification system in predicting 1-, 2-, 3-, 4-, and 5-year PFI were 0.687 (95\% CI, 0.592-0.782), 0.620 (95\% CI, 0.537-0.702), 0.613 (95\% CI, $0.534-0.692), 0.647$ (95\% CI, 0.564-0.730), and 0.625 (95\% CI, 0.538-0.712), respectively. The AUCs of the MACIS score in predicting 1-, 2-, 3-, 4-, and 5-year PFI were 0.724 (95\% CI, 0.594-0.854), 0.638 (95\% CI, 0.517-0.722), 0.687 (95\% CI, $0.587-0.787), 0.716$ (95\% CI, $0.621-0.811)$, and 0.694 (95\% CI, 0.587-0.801), respectively. The AUCs of the AJCC staging system in predicting 1-, 2-, 3-, 4-, and 5-year PFI were 0.657 (95\% CI, 0.514-0.800), 0.628 (95\% CI, 0.520-0.735), 0.691 (95\% CI, 0.534-0.692), 0.732 (95\% CI, 0.629-0.834), and 0.707 (95\% CI, 0.590-0.824), respectively. The nomogram had significantly higher AUCs in predicting 1-, 2-, 3-, 4-, and 5-year PFI in comparison with the ATA risk stratification system and MACIS score and had significantly higher AUCs in predicting 1-, 2-, and 3-year PFI in comparison with the AJCC staging system $(P<0.05)$.

\section{DISCUSSION}

Accumulating evidence has shown that circRNAs and their mediated ceRNA regulatory networks play a vital role in pathogenesis and progression of various human cancers, including PTC. hsa-circ-0058124 was previously identified to be upregulated in PTC and associated with poor prognosis. By sponging hsa-miR-218-5p, hsa-circ-0058124 promoted proliferation, invasion, and metastasis of PTC via the NOTCH3/GATAD2A signaling axis (Yao et al., 2019). Through regulation of $A B C A 9$ and MTA1 via sponge hsa-miR-1179 and hsa-miR-1205, upregulated hsa_circ_0039411 promoted proliferation, migration, and invasion of PTC cells and inhibited cell apoptosis (Yang et al., 2020). CircRNAs are resistant to exonase and RNase $\mathrm{R}$, enabling them to be more stable than other types of ncRNAs, as well as play an important regulatory role in cancer cell. The current understanding of the circRNA-related ceRNA network in PTC is limited, requiring further study.

In this study, DE-circRNAs, DE-miRNAs, and DE-mRNAs with potential ceRNA regulatory relationships in PTC were identified through comprehensive analysis of targeting relationship prediction and differential expression data. Based on the further correlation analysis of expression between DE-miRNAs and DE-mRNAs with potential ceRNA regulatory relationships, a circRNA-miRNA-mRNA regulatory network was constructed in PTC. DE-mRNAs within the ceRNA network associated with PFI of PTC were further identified to construct a ceRNA regulatory subnetwork associated with PFI. Six hub DEmRNAs, namely, CLCNKB, FXBO27, FXYD6, RIMS2, SPC24, and $C D K N 2 A$, were identified to be most significantly related to the PFI of PTC, which were regulated by three DE-miRNAs, including hsa-miR-146b-3p, hsa-miR-139-5p, and hsa-miR139-3p. They were subsequently regulated by $11 \mathrm{DE}$-circRNAs via the ceRNA mechanism, including hsa_circ_0003645, hsa_circ_0089153, hsa_circ_0005699, hsa_circ_0007146, hsa_circ_0038718, hsa_circ_0001658, hsa_circ_0008784, hsa_circ_0000965, hsa_circ_0001917, hsa_circ_0008354, and hsa_circ_0049271. These newly identified key circRNA-miRNAmRNA regulatory relationships may help elucidate the molecular mechanism of progression in post-surgical PTC. Such insights may provide novel therapeutic targets for treatment of recurrence in PTC. Hub DE-mRNAs identified in the ceRNA network were potential predictors of PFI in PTC.

Although most patients with PTC have a relatively good prognosis, a portion of patients will eventually develop postsurgical recurrence. These high-risk PTC patients should be distinguished early enough to adopt more aggressive treatment options, additional adjuvant radioactive iodine therapy, thyroid 
TABLE 3 | Baseline characteristics of patients included for the evaluation of prognostic factors and establishment of nomogram.

\begin{tabular}{|c|c|c|c|}
\hline \multirow[t]{2}{*}{ Clinical features } & Low risk & High risk & \multirow[t]{2}{*}{$P$-value } \\
\hline & \multicolumn{2}{|c|}{ Mean $\pm S D$} & \\
\hline Follow-up time (day) & $1,224.96 \pm 968.27$ & $1,297.97 \pm 925.63$ & 0.570 \\
\hline DE-mRNA signature & $0.18 \pm 0.37$ & $0.97 \pm 0.27$ & $<0.001$ \\
\hline \multirow[t]{2}{*}{ Age } & $46.54 \pm 14.69$ & $49.93 \pm 18.9$ & 0.102 \\
\hline & \multicolumn{2}{|c|}{$n(\%)$} & \\
\hline PFI & & & $<0.001$ \\
\hline Progression-free & 300 (93.46\%) & 49 (72.06\%) & \\
\hline Progression & $21(6.54 \%)$ & 19 (27.94\%) & \\
\hline BRAF V600E & & & 0.026 \\
\hline Wild type & 121 (37.69\%) & 16 (23.53\%) & \\
\hline Mutant & $200(62.31 \%)$ & 52 (76.47\%) & \\
\hline RAS mutation & & & 0.364 \\
\hline Wild type & 280 (87.23\%) & $62(91.18 \%)$ & \\
\hline Mutant & $41(12.77 \%)$ & $6(8.82 \%)$ & \\
\hline RET mutation & & & 0.293 \\
\hline Wild type & $295(91.90 \%)$ & 65 (95.59\%) & \\
\hline Mutant & $26(8.10 \%)$ & $3(4.41 \%)$ & \\
\hline NTRK1 mutation & & & 0.256 \\
\hline Wild type & 315 (98.13\%) & 68 (100.00\%) & \\
\hline Mutant & $6(1.87 \%)$ & 0 (0.00\%) & \\
\hline TERT mutation & & & 0.182 \\
\hline Wild type & $318(99.07 \%)$ & 66 (97.06\%) & \\
\hline Mutant & $3(0.93 \%)$ & $2(2.94 \%)$ & \\
\hline Sex & & & 0.180 \\
\hline Male & $83(25.86 \%)$ & $23(33.82 \%)$ & \\
\hline Female & 238 (74.14\%) & 45 (66.18\%) & \\
\hline Histological type & & & 0.303 \\
\hline $\begin{array}{l}\text { Thyroid papillary } \\
\text { carcinoma- } \\
\text { classical/usual }\end{array}$ & 226 (70.40\%) & $52(76.47 \%)$ & \\
\hline $\begin{array}{l}\text { Thyroid papillary } \\
\text { carcinoma-follicular } \\
\text { ( } \geq 99 \% \text { follicular } \\
\text { patterned) }\end{array}$ & 67 (20.87\%) & $8(11.76 \%)$ & \\
\hline $\begin{array}{l}\text { Thyroid papillary } \\
\text { carcinoma-tall cell } \\
\text { ( } \geq 50 \% \text { tall cell } \\
\text { features) }\end{array}$ & 22 (6.85\%) & 7 (10.29\%) & \\
\hline Others & $6(1.87 \%)$ & $1(1.47 \%)$ & \\
\hline $\mathbf{T}$ & & & $<0.001$ \\
\hline $\mathrm{T} 1$ & 107 (33.33\%) & $6(8.82 \%)$ & \\
\hline T2 & 104 (32.40\%) & 20 (29.41\%) & \\
\hline T3 & 103 (32.09\%) & 32 (47.06\%) & \\
\hline $\mathrm{T} 4$ & 7 (2.18\%) & 10 (14.71\%) & \\
\hline $\mathbf{N}$ & & & $<0.001$ \\
\hline NO & 175 (54.52\%) & 18 (26.47\%) & \\
\hline N1 & 39 (12.15\%) & 16 (23.53\%) & \\
\hline $\mathrm{N} 1 \mathrm{a}$ & 63 (19.63\%) & 16 (23.53\%) & \\
\hline N1b & 44 (13.71\%) & 18 (26.47\%) & \\
\hline M & & & 0.040 \\
\hline $\mathrm{MO}$ and $\mathrm{Mx}$ & 199 (61.99\%) & 33 (48.53\%) & \\
\hline M1 & 122 (38.01\%) & 35 (51.47\%) & \\
\hline
\end{tabular}

(Continued)
TABLE 3 | Continued

\begin{tabular}{|c|c|c|c|}
\hline \multirow[t]{2}{*}{ Clinical features } & Low risk & High risk & \multirow[t]{2}{*}{$P$-value } \\
\hline & \multicolumn{2}{|c|}{ Mean $\pm S D$} & \\
\hline AJCC stage & & & $<0.001$ \\
\hline Stage I & 188 (58.57\%) & $28(41.18 \%)$ & \\
\hline Stage II & 37 (11.53\%) & 2 (2.94\%) & \\
\hline Stage III & 72 (22.43\%) & $18(26.47 \%)$ & \\
\hline Stage IV & 24 (7.48\%) & 20 (29.41\%) & \\
\hline Residual tumor & & & 0.073 \\
\hline Ro & 265 (82.55\%) & 48 (70.59\%) & \\
\hline $\mathrm{Rx}$ & 23 (7.17\%) & 10 (14.71\%) & \\
\hline $\mathrm{R} 1$ & 30 (9.35\%) & $10(14.71 \%)$ & \\
\hline $\mathrm{R} 2$ & 3 (0.93\%) & 0 (0.00\%) & \\
\hline $\begin{array}{l}\text { Extrathyroid } \\
\text { extension }\end{array}$ & & & $<0.001$ \\
\hline None & 228 (71.03\%) & 35 (51.47\%) & \\
\hline Minimal (T3) & 87 (27.10\%) & 25 (36.76\%) & \\
\hline $\begin{array}{r}\text { Moderate or } \\
\text { advanced (T4) }\end{array}$ & $6(1.87 \%)$ & $8(11.76 \%)$ & \\
\hline Multifocality & & & 0.006 \\
\hline Unifocal & 163 (50.78\%) & 47 (69.12\%) & \\
\hline Multifocal & $158(49.22 \%)$ & 21 (30.88\%) & \\
\hline Anatomic site & & & 0.585 \\
\hline Unilateral & $248(77.26 \%)$ & 56 (82.35\%) & \\
\hline Isthmus & $17(5.30 \%)$ & 2 (2.94\%) & \\
\hline Bilateral & $56(17.45 \%)$ & $10(14.71 \%)$ & \\
\hline
\end{tabular}

hormone suppression therapy with a higher dosage, and timely intervention to prevent post-surgical progression if necessary. In contrast, for the remaining low-risk PTC patients, aggressive treatment options should be avoided to improve quality of life. Therefore, it is critical to accurately predict the post-surgical risk of progression in patients with PTC. Currently, the ATA guidelines recommend the use of AJCC staging system and MACIS score to predict the risk of post-operative mortality. ATA risk stratification system was recommended to assess postsurgical risk of recurrence. The existing risk assessment tools are not sufficiently accurate, and a novel prediction system should be established to more accurately predict the prognosis of PTC patients. In the current study, we established a circRNAassociated ceRNA network in the PTC. Based on this, six hub PFI-related DE-mRNAs of the PTC were identified. A six-DEmRNA signature was subsequently established, which was able to distinguish high-risk PTC patients from the low-risk ones and could accurately predict the PFI. Nomogram has been widely applied in oncology to assess the prognosis of cancer patients (Balachandran et al., 2015). Nomogram can integrate various prognostic factors, including molecular and clinical parameters, and provide a visual graphical interface for personalized prediction of clinical events. In this study, to establish a more accurate assessment tool for prognosis evaluation in post-surgical PTC, a prognostic nomogram was established incorporating the DE-mRNA signature and clinicopathological parameters. This 
TABLE 4 | Unadjusted univariate Cox analysis.

\begin{tabular}{|c|c|c|}
\hline Clinical features & Statistics & PFI \\
\hline DE-mRNA signature & $0.32 \pm 0.46$ & $6.90(3.45,13.79),<0.000$ \\
\hline \multicolumn{3}{|l|}{ BRAF V6OOE } \\
\hline Wild type & 137 (35.22\%) & 1.0 \\
\hline Mutant & $252(64.78 \%)$ & $1.06(0.55,2.06), 0.860$ \\
\hline \multicolumn{3}{|l|}{ RAS mutation } \\
\hline Wild type & 342 (87.92\%) & 1.0 \\
\hline Mutant & $47(12.08 \%)$ & $1.72(0.76,3.89), 0.194$ \\
\hline \multicolumn{3}{|l|}{ TERT mutation } \\
\hline Wild type & $384(98.71 \%)$ & 1.0 \\
\hline Mutant & $5(1.29 \%)$ & 0.00 (0.00, Inf), 0.997 \\
\hline \multicolumn{3}{|l|}{ RET mutation } \\
\hline Wild type & 360 (92.54\%) & 1.0 \\
\hline Mutant & $29(7.46 \%)$ & $0.72(0.17,3.00), 0.657$ \\
\hline \multicolumn{3}{|l|}{ NTRK1 mutation } \\
\hline Wild type & $383(98.46 \%)$ & 1.0 \\
\hline Mutant & $6(1.54 \%)$ & 0.00 (0.00, Inf), 0.996 \\
\hline \multicolumn{3}{|l|}{ Sex } \\
\hline Male & $106(27.25 \%)$ & 1.0 \\
\hline Female & $283(72.75 \%)$ & $0.76(0.39,1.47), 0.411$ \\
\hline Age & $47.13 \pm 15.54$ & $1.02(1.00,1.04), 0.042$ \\
\hline \multicolumn{3}{|l|}{ Age } \\
\hline$\leq 55$ years & 275 (70.69\%) & 1.0 \\
\hline$>55$ years & $114(29.31 \%)$ & $2.36(1.27,4.41), 0.007$ \\
\hline \multicolumn{3}{|l|}{ Histological type } \\
\hline $\begin{array}{l}\text { Thyroid papillary } \\
\text { carcinoma- } \\
\text { classical/usual }\end{array}$ & $278(71.47 \%)$ & 1.0 \\
\hline $\begin{array}{l}\text { Thyroid papillary } \\
\text { carcinoma-follicular } \\
\text { ( } \geq 99 \% \text { follicular } \\
\text { patterned) }\end{array}$ & 75 (19.28\%) & $0.72(0.28,1.87), 0.501$ \\
\hline $\begin{array}{l}\text { Thyroid papillary } \\
\text { carcinoma-tall cell } \\
\text { ( } \geq 50 \% \text { tall cell } \\
\text { features) }\end{array}$ & $29(7.46 \%)$ & $2.52(1.04,6.08), 0.041$ \\
\hline Others & $7(1.80 \%)$ & 0.00 (0.00, Inf), 0.997 \\
\hline \multicolumn{3}{|l|}{ Aggressive subtype } \\
\hline No & 359 (92.29\%) & 1.0 \\
\hline Yes & $30(7.71 \%)$ & $2.70(1.13,6.46), 0.025$ \\
\hline \multicolumn{3}{|l|}{$\mathbf{T}$} \\
\hline $\mathrm{T} 1$ & 113 (29.05\%) & 1.0 \\
\hline T2 & $124(31.88 \%)$ & $2.63(0.85,8.16), 0.094$ \\
\hline Т3 & $135(34.70 \%)$ & $4.22(1.44,12.36), 0.009$ \\
\hline T4 & $17(4.37 \%)$ & $5.73(1.43,22.98), 0.014$ \\
\hline \multicolumn{3}{|l|}{$\mathbf{N}$} \\
\hline NO & $193(49.61 \%)$ & 1.0 \\
\hline N1 & 196 (50.39\%) & 2.11 (1.09, 4.08), 0.027 \\
\hline \multicolumn{3}{|l|}{ M } \\
\hline $\mathrm{MO}$ and $\mathrm{Mx}$ & 232 (59.64\%) & 1.0 \\
\hline M1 & 157 (40.36\%) & $1.65(0.89,3.08), 0.112$ \\
\hline \multicolumn{3}{|l|}{ AJCC stage } \\
\hline Stage I & 216 (55.53\%) & 1.0 \\
\hline Stage II & 39 (10.03\%) & $1.13(0.32,3.93), 0.849$ \\
\hline
\end{tabular}

(Continued)
TABLE $4 \mid$ Continued

\begin{tabular}{|c|c|c|}
\hline Clinical features & Statistics & PFI \\
\hline Stage III & $90(23.14 \%)$ & $2.67(1.27,5.60), 0.010$ \\
\hline Stage IV & $44(11.31 \%)$ & $3.89(1.68,9.02), 0.002$ \\
\hline \multicolumn{3}{|l|}{ Residual tumor } \\
\hline Ro & $313(80.46 \%)$ & 1.0 \\
\hline$R x$ & $33(8.48 \%)$ & $1.76(0.61,5.01), 0.293$ \\
\hline $\mathrm{R} 1$ & $40(10.28 \%)$ & $1.74(0.72,4.18), 0.219$ \\
\hline $\mathrm{R} 2$ & $3(0.77 \%)$ & $3.23(0.44,23.91), 0.251$ \\
\hline \multicolumn{3}{|c|}{ Extrathyroid extension } \\
\hline None & $263(67.61 \%)$ & 1.0 \\
\hline Minimal (T3) & $112(28.79 \%)$ & $1.85(0.97,3.50), 0.060$ \\
\hline $\begin{array}{l}\text { Moderate or } \\
\text { advanced (T4) }\end{array}$ & $14(3.60 \%)$ & $1.77(0.42,7.58), 0.439$ \\
\hline $\begin{array}{l}\text { Neoplasm largest } \\
\text { dimension }(\mathrm{cm})\end{array}$ & $2.80 \pm 1.58$ & $1.24(1.04,1.46), 0.014$ \\
\hline \multicolumn{3}{|c|}{ Neoplasm largest dimension } \\
\hline$\leq 2 \mathrm{~cm}$ & $153(39.33 \%)$ & 1.0 \\
\hline$>2 \mathrm{~cm}$ & $236(60.67 \%)$ & $3.61(1.51,8.61), 0.004$ \\
\hline \multicolumn{3}{|l|}{ Multifocality } \\
\hline Unifocal & $210(53.98 \%)$ & 1.0 \\
\hline Multifocal & $179(46.02 \%)$ & $0.82(0.43,1.57), 0.553$ \\
\hline \multicolumn{3}{|l|}{ Anatomic site } \\
\hline Unilateral & 304 (78.15\%) & 1.0 \\
\hline Isthmus & $19(4.88 \%)$ & $0.45(0.06,3.27), 0.429$ \\
\hline Bilateral & 66 (16.97\%) & $0.94(0.39,2.26), 0.898$ \\
\hline
\end{tabular}

nomogram was able to accurately predict PFI of PTC and was better than the ATA risk stratification system, MACIS score, and AJCC staging system recommended by ATA guidelines.

Currently, 11 DE-circRNAs were identified to regulate the six hub DE-mRNAs via the ceRNA mechanism in the study. The role of hsa_circ_0089153 in PTC has previously been reported. hsa_circ_0089153 was upregulated in clinical specimens of PTC (Li et al., 2018). In vitro experiments indicated that downregulation of hsa_circ_0089153 expression could inhibit proliferation, migration, and invasion of PTC cells. The luciferase reporter assay confirmed that hsa_circ_0089153 sponged hsamiR-145 to mediate upregulation of ZEB2 expression, thereby playing a carcinogenic role in PTC. hsa_circ_0089153 was also reported to be upregulated in gastric cancer and bladder cancer (Wei et al., 2019; Zhuang et al., 2020). Our study indicated that upregulated hsa_circ_0089153 may sponge hsa-miR-139-3p and upregulated SPC24 and CDKN2A expression to promote PTC progression. hsa_circ_0003645 was previously identified to be upregulated in non-small cell lung cancer tissue (Zhang et al., 2018). hsa_circ_0038718 was reported to be upregulated in hepatocellular carcinoma

(Qiu et al., 2019). Our result suggested that hsa_circ_0003645 and hsa_circ_0038718 may also play an oncogenic role as a sponge of hsa-miR-139-3p. The function of hsa_circ_0001917 in PTC has not been reported. However, hsa_circ_0001917 was also identified to be upregulated in hepatocellular carcinoma (Qiu et al., 2019). Our result suggested that the upregulated 
TABLE 5 | Multivariate Cox regression analysis.

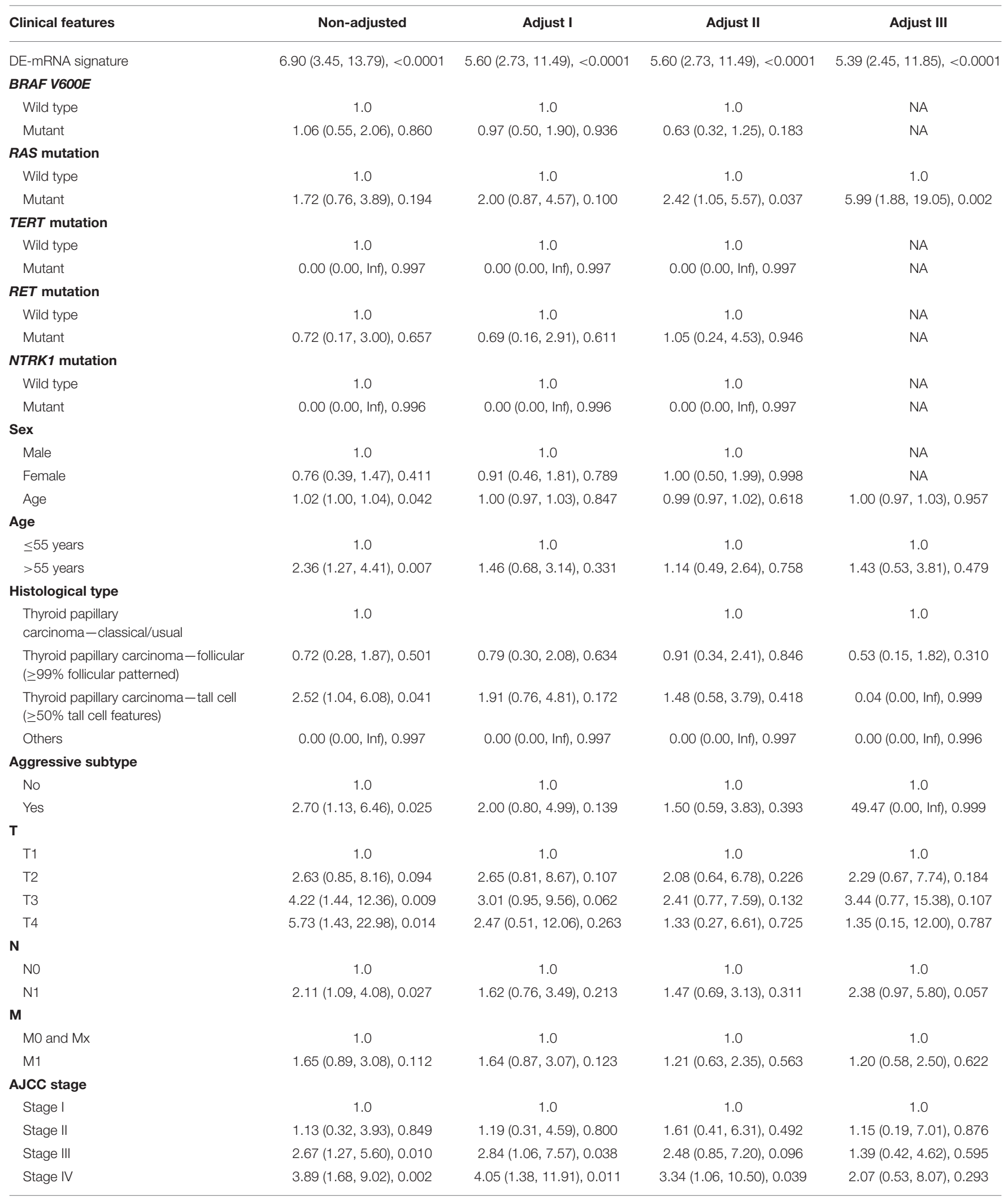


TABLE 5 | Continued

\begin{tabular}{|c|c|c|c|c|}
\hline Clinical features & Non-adjusted & Adjust I & Adjust II & Adjust III \\
\hline \multicolumn{5}{|l|}{ Residual tumor } \\
\hline RO & 1.0 & 1.0 & 1.0 & 1.0 \\
\hline$R x$ & $1.76(0.61,5.01), 0.293$ & $1.35(0.47,3.93), 0.579$ & $1.25(0.43,3.64), 0.684$ & $1.56(0.48,5.06), 0.462$ \\
\hline $\mathrm{R} 1$ & $1.74(0.72,4.18), 0.219$ & $1.20(0.49,2.96), 0.691$ & $1.14(0.46,2.84), 0.776$ & $1.17(0.44,3.12), 0.748$ \\
\hline $\mathrm{R} 2$ & $3.23(0.44,23.91), 0.251$ & 5.58 (0.47, 66.80), 0.174 & $4.15(0.33,52.73), 0.272$ & $11.00(0.78,154.44), 0.075$ \\
\hline Minimal (T3) & $1.85(0.97,3.50), 0.060$ & $1.30(0.64,2.61), 0.465$ & $1.23(0.62,2.47), 0.553$ & $0.71(0.24,2.10), 0.533$ \\
\hline Moderate or advanced (T4) & $1.77(0.42,7.58), 0.439$ & 0.68 (0.14, 3.30), 0.632 & $0.46(0.09,2.23), 0.332$ & $0.83(0.09,7.48), 0.865$ \\
\hline Neoplasm largest dimension & $1.24(1.04,1.46), 0.014$ & $1.14(0.96,1.36), 0.140$ & $1.04(0.86,1.25), 0.686$ & $0.95(0.76,1.20), 0.691$ \\
\hline \multicolumn{5}{|l|}{ Neoplasm largest dimension } \\
\hline$\leq 2 \mathrm{~cm}$ & 1.0 & 1.0 & 1.0 & 1.0 \\
\hline Multifocal & $0.82(0.43,1.57), 0.553$ & $0.72(0.37,1.39), 0.322$ & $0.92(0.47,1.77), 0.792$ & NA \\
\hline \multicolumn{5}{|l|}{ Anatomic site } \\
\hline Unilateral & 1.0 & 1.0 & 1.0 & NA \\
\hline Isthmus & $0.45(0.06,3.27), 0.429$ & $0.48(0.06,3.54), 0.472$ & 0.41 (0.06, 3.00), 0.377 & NA \\
\hline Bilateral & 0.94 (0.39, 2.26), 0.898 & 0.73 (0.30, 1.77), 0.486 & $0.88(0.36,2.14), 0.782$ & NA \\
\hline
\end{tabular}

Adjust I model adjusted for age, sex, and AJCC stage.

Adjust II model adjusted for age, sex, AJCC stage, and DE-mRNA signature.

Adjust III model adjusted for parameters with $P<0.25$ based on univariate analysis.

hsa_circ_0001917 may sponge hsa-miR-139-5p, promoting PTC via upregulation of RIMS2. The function of hsa_circ_0005699 in cancer was controversial. hsa_circ_0005699 was previously reported to downregulate $M C M 8$ and NCAPD2 expression by acting as a sponge of hsa-miR-504, functioning as tumor suppressor in gastric cancer (Guan et al., 2019). Our study suggested that hsa_circ_0005699 may promote PTC as a sponge of hsa-miR-139-3p and upregulate SPC24 and CDKN2A expression. The function of the remaining six circRNAs in cancer is still known. Our study suggests that the upregulation of hsa_circ_0007146 may promote PTC through the sponging of hsa-miR-139-3p. hsa_circ_0001658, hsa_circ_0008784, and hsa_circ_0000965 may upregulate RIMS2 expression as a sponge of hsa-miR-139-5p, playing an oncogenic role in PTC. Moreover, downregulated hsa_circ_0008354 and hsa_circ_0049271 may sponge hsa-miR-146b-3p and suppress the expression of CLCNKB, FBXO27, and FXYD6. The function of these circRNAs in PTC requires further experimental validation.

Six hub DE-mRNAs markedly associated with the PFI of PTC were identified in the current study. Upregulated SPC24 and CDKN2A were identified as targets of hsa-miR-139$3 p$. The tumor-suppressing role of hsa-miR-139-3p has been identified in multiple tumors (Sannigrahi et al., 2017). SPC24 is an important component of kinetochore-associated NDC80 complex. This complex mediates chromosome segregation and spindle checkpoint activity. SPC24 plays an important role in maintaining the integrity of kinetochore (McCleland et al., 2004). SPC24 was previously identified to be highly expressed in anaplastic thyroid cancer. Knockdown of SPC24 expression inhibited cell growth and invasion and promoted tumor cell apoptosis (Yin et al., 2017). SPC24 was also reported to be highly expressed in hepatocellular carcinoma and was an independent predictor of survival (Zhu et al., 2015). Downregulation of SPC24 inhibited growth and invasion of tumor cells and promoted apoptosis. The oncogenic role of SPC24 was also identified in breast cancer and lung cancer (Zhou et al., 2017, 2018). Here, SPC24 was identified to be negatively regulated by hsa-miR-139-3p. This regulatory role between SPC24 and hsa-miR-139-3p was previously observed in bladder cancer (Yonemori et al., 2016). CDKN2A is traditionally known as a tumor-suppressor gene coding for two proteins, including the p16INK4a and p14arf (Bockstaele et al., 2006). CDKN2A is involved in cell cycle regulation. However, mounting data suggest that CDKN2A may play a dual role in multiple tumors. The p14arf that it codes plays an important role in invasion and metastasis and is associated with a poor prognosis (Fontana et al., 2019). Upregulation of p14arf has been identified in multiple hematological malignancies, aggressive types of Bcell lymphomas, and bladder cancers (Sánchez-Aguilera et al., 2002; Berggren et al., 2003; Lee et al., 2003). The oncogenic function of p14arf is associated with the autophagy regulation (Fontana et al., 2019). Downregulation of p14arf was shown to inhibit progression of lymphomas with the MYC mutation (Humbey et al., 2008). Particularly in PTC, p16INK4a, and p14arf coded by $C D K N 2 A$ were both identified to be upregulated in thyroid tumorigenesis (Ferru et al., 2006). Wild-type p14arf has 
A

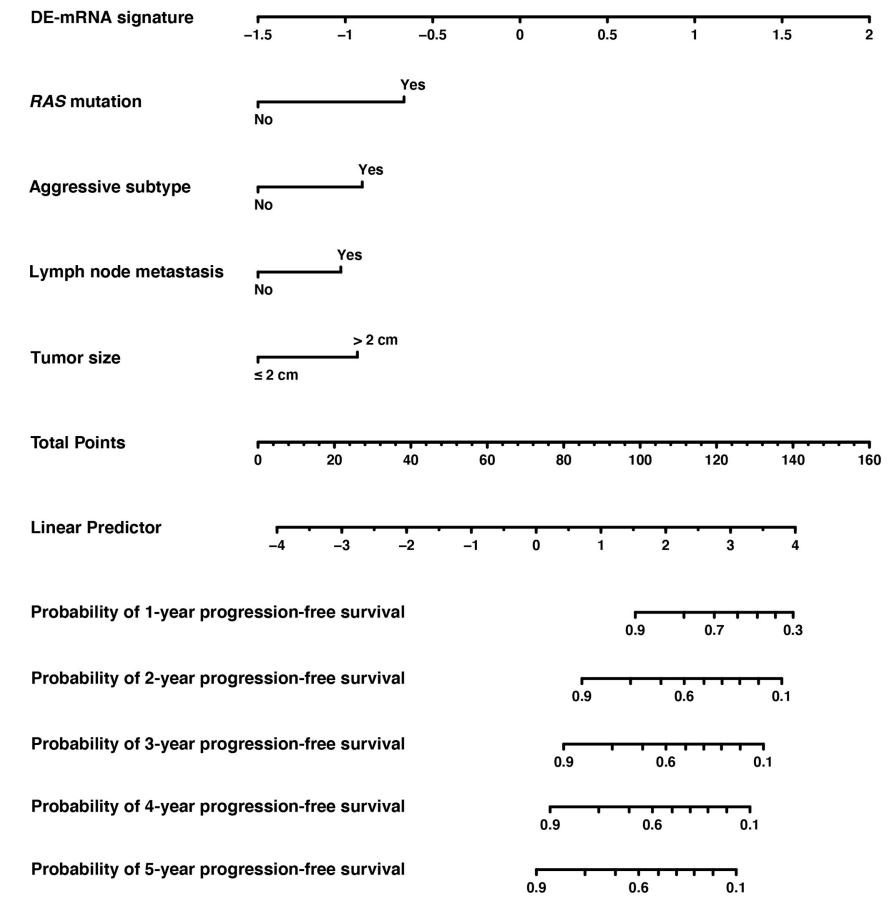

D
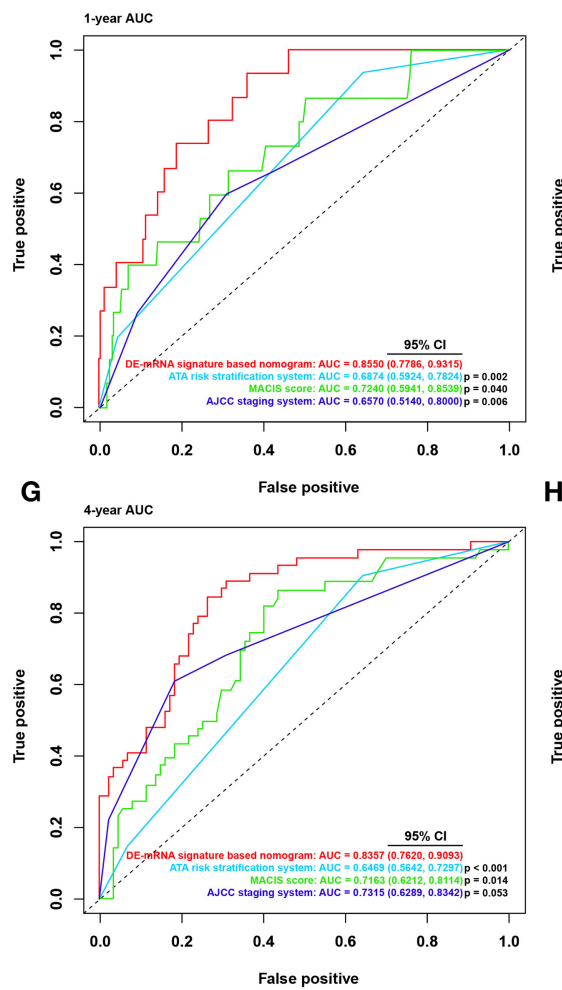

E

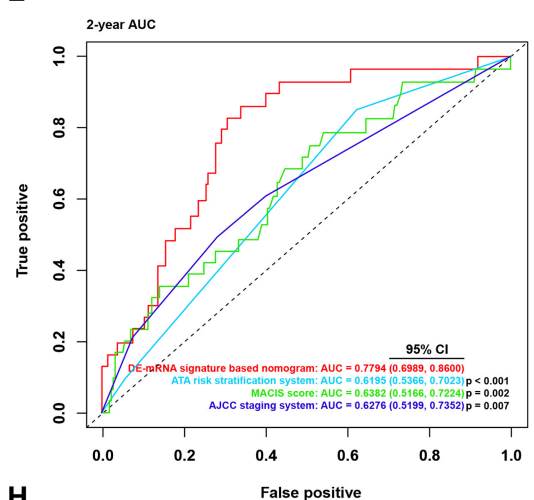

H

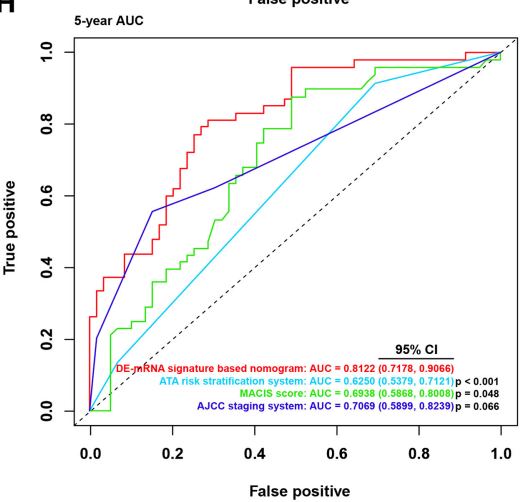

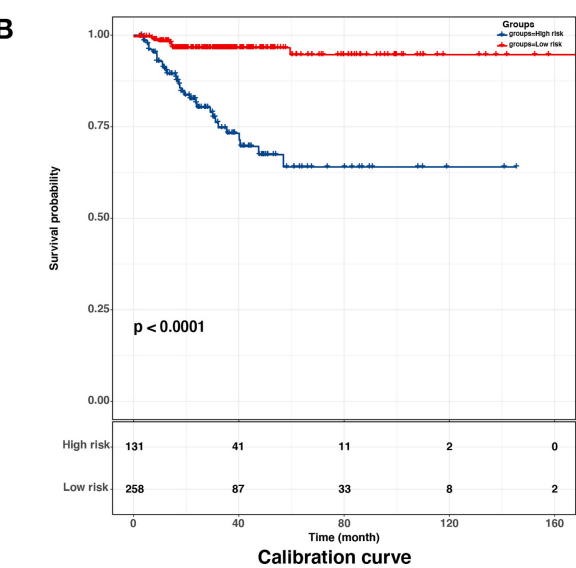

C
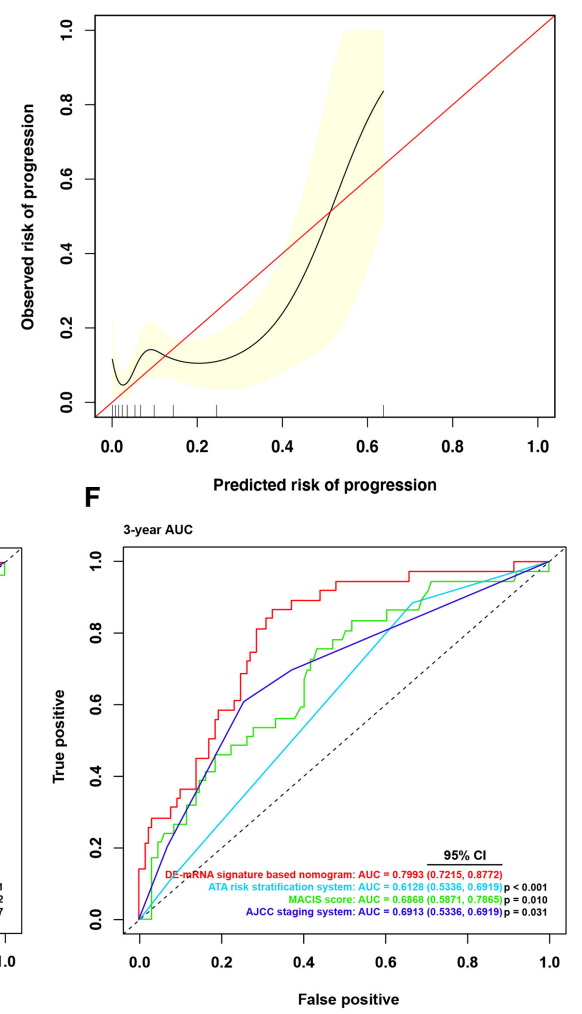

FIGURE 8 | Building and validation of a DE-mRNA signature-based prognostic nomogram predicting PFI in PTC. (A) A DE-mRNA signature-based prognostic nomogram predicting 1-, 2-, 3-, 4-, and 5-year PFI in PTC. (B) The Kaplan-Meier curves for the nomogram based on the optimal cutoff value in the entire 
FIGURE 8 | TCGA-THCA dataset. The horizontal axis shows the PFI time in months, and the vertical axis shows the probability of progression-free survival. (C) The calibration plot for internal validation of the nomogram. The $X$ axis represents the predicted risk of progression, whereas the $Y$ axis represents the observed risk of progression. (D) The time-dependent ROC curves for 1-year PFI predicted by the nomogram in comparison with the ATA risk stratification system, the MACIS score, and the AJCC staging system. (E) The time-dependent ROC curves for 2-year PFI predicted by the nomogram in comparison with the ATA risk stratification system, the MACIS score, and the AJCC staging system. (F) The time-dependent ROC curves for 3-year PFI predicted by the nomogram in comparison with the ATA risk stratification system, the MACIS score, and the AJCC staging system. (G) The time-dependent ROC curves for 4-year PFI predicted by the nomogram in comparison with the ATA risk stratification system, the MACIS score, and the AJCC staging system. (H) The time-dependent ROC curves for 5-year PFI predicted by the nomogram in comparison with the ATA risk stratification system, the MACIS score, and the AJCC staging system. AUCs were compared with the method described by Subramanian et al. (2005).

been observed to delocalize into the cytoplasm in aggressive PTC. Here, we further identified that $C D K N 2 A$ was upregulated in PTC and was associated with shorter PFI, and hsa-miR139-3p was the potential negative regulator of CDKN2A. The function of CDKN2A in PTC progression requires further study. RIMS2 was also identified to be upregulated in PTC and was potentially regulated by hsa-miR-139-5p. hsa-miR-139$5 \mathrm{p}$ was identified to be downregulated in the primary tumor and further in PTC metastasis (Montero-Conde et al., 2020). hsa-miR-139-5p was also able to be sponged by circBACH2 and relieved the suppression of the target gene LMO4 in PTC (Cai et al., 2019). RIMS2 was identified as a novel target of hsa-miR-139-5p in this study. RIMS2 is a Rab effector and scaffold protein associated with exocytosis (Yoo et al., 2013). It was recently reported to have a high mutation rate in melanoma and mantle cell lymphoma (Hill et al., 2020; Zhang and Xia, 2020). In this study, RIMS2 was identified as a hub DE-mRNA in the ceRNA network and was associated with PFI of PTC. The function of RIMS2 in PTC requires further experimental validation.

In this study, downregulated CLCNKB, FBXO27, and FXYD6 were identified to be novel targets of hsa-miR-146b-3p in PTC. hsa-miR-146b-3p was previously reported to be upregulated in PTC and positively associated with central lymph node metastases (Han et al., 2016). hsa-miR-146b-3p may promote invasion and metastasis of PTC by targeting NF2 (Yu et al., 2018). hsa-miR-146b-3p also targeted PAX8 in modulating the differentiated phenotype of PTC (Riesco-Eizaguirre et al., 2015). FXYD6 is known as a specific modulator of $\mathrm{Na}$ and K-ATPase and is expressed in multiple epithelial cells of the inner ear (Delprat et al., 2007). In accordance with the current study, FXYD6 was previously identified to be downregulated in PTC and was associated with poor prognosis (Wu et al., 2019). $C L C N K B$ is a voltage-gated chloride channel participating in the regulation of cell volume, membrane potential stabilization, signal transduction, and transepithelial transport (Estévez et al., 2001). CLCNKB was previously reported to be downregulated in renal carcinoma (Murakami et al., 2007). Hypermethylation in deletions of $C L C N K B$ in renal carcinoma further indicated its tumor-suppressing role in cancer (Girgis et al., 2012). FBXO27 is a component for substrate recognition in the SCF-type E3 ubiquitin ligase complex (Glenn et al., 2008). Its role in cancer is currently unknown. In this study, CLCNKB, FBXO27, and FXYD6 were identified to be negatively regulated by hsa-miR-146b-3p. Together, hsa_circ_0008354 and hsa_circ_0049271 formed an important part of the PFI-related
ceRNA subnetwork we established. The potential tumorsuppressing role of CLCNKB, FBXO27, and FXYD6 in PTC requires further validation.

To the best of our knowledge, the ceRNA network we established and the prognostic DE-mRNA signature we proposed have not been reported previously. The nomogram incorporating the DE-mRNA signature and clinical parameters was robust in predicting PFI of PTC. DE-circRNAs, DEmiRNAs, and DE-mRNAs were potential therapeutic targets for prevention and treatment of recurrent PTC. We acknowledge that our study inevitably has some limitations. First, sequencing data and follow-up data of PTCs in our study were based on TCGA-THCA dataset. Most patients were from North America. Therefore, caution should be exercised in extrapolating the conclusions from findings to other populations. Second, the targeting relationship of ceRNA is based on bioinformatics speculation and requires further experimental validation. Finally, the biological function of certain DE-circRNAs, DE-miRNAs, and DE-mRNAs requires investigation with experiments in PTC.

\section{CONCLUSIONS}

This study revealed a circRNA-associated ceRNA network in PTC. Based on survival analysis, a ceRNA subnetwork associated with PFI in PTC was identified. The molecules within the PFI-related subnetwork may represent a promising target for the treatment of patients with recurrent PTC. With the hub DE-mRNAs identified within the subnetwork, a prognostic six-DE-mRNA signature was established. The nomogram incorporating the DE-mRNA signature and clinical parameters is robust in predicting the PFI of post-surgical PTC.

\section{DATA AVAILABILITY STATEMENT}

Publicly available datasets were analyzed in this study. This data can be found here: the datasets analyzed during the current study are available in the Gene Expression Omnibus (https://www.ncbi. nlm.nih.gov/geo/) and the Cancer Genome Atlas (https://portal. gdc.cancer.gov/).

\section{AUTHOR CONTRIBUTIONS}

ZL, XL, and XX: conception and design. MW, SL, JH, and RL: development of methodology. MW and HY: analysis 
and interpretation of data. MW: writing of the manuscript. $\mathrm{XX}, \mathrm{SL}, \mathrm{JH}$, and XL: review of the manuscript. ZL: study supervision. All authors contributed to the article and approved the submitted version.

\section{FUNDING}

This research was supported by the Nature Science Foundation of Beijing [grant number: 7202164], CAMS Innovation Fund for Medical Sciences (CIFMS) [grant number: 2016-12M-3005], and CAMS Innovation Fund for graduate students [grant number: 2019-1002-44].

\section{REFERENCES}

Antonelli, A., and La Motta, C. (2017). Novel therapeutic clues in thyroid carcinomas: The role of targeting cancer stem cells. Med. Res. Rev. 37, 1299-1317. doi: 10.1002/med.21448

Balachandran, V. P., Gonen, M., Smith, J. J., and DeMatteo, R. P. (2015). Nomograms in oncology: more than meets the eye. Lancet Oncol. 16, e173e180. doi: 10.1016/S1470-2045(14)71116-7

Berggren, P., Kumar, R., Sakano, S., Hemminki, L., Wada, T., Steineck, G., et al. (2003). Detecting homozygous deletions in the CDKN2A(p16(INK4a))/ARF(p14(ARF)) gene in urinary bladder cancer using real-time quantitative PCR. Clin. Cancer Res. 9, 235-242.

Biondi, B., and Cooper, D. S. (2018). Subclinical hyperthyroidism. N. Engl. J. Med. 378, 2411-2419. doi: 10.1056/NEJMcp1709318

Blanche, P., Dartigues, J. F., and Jacqmin-Gadda, H. (2013). Estimating and comparing time-dependent areas under receiver operating characteristic curves for censored event times with competing risks. Stat. Med. 32, 5381-5397. doi: 10.1002/sim.5958

Bockstaele, L., Kooken, H., Libert, F., Paternot, S., Dumont, J. E., de Launoit, Y., et al. (2006). Regulated activating Thr172 phosphorylation of cyclin-dependent kinase 4(CDK4): its relationship with cyclins and CDK "inhibitors". Mol. Cell. Biol. 26, 5070-5085. doi: 10.1128/MCB.02006-05

Cabanillas, M. E., McFadden, D. G., and Durante, C. (2016). Thyroid cancer. Lancet. 388, 2783-2795. doi: 10.1016/S0140-6736(16)30172-6

Cai, X., Zhao, Z., Dong, J., Lv, Q., Yun, B., Liu, J., et al. (2019). Circular RNA circBACH2 plays a role in papillary thyroid carcinoma by sponging miR-139-5p and regulating LMO4 expression. Cell Death Dis. 10:184. doi: 10.1038/s41419-019-1439-y

Camp, R. L., Dolled-Filhart, M., and Rimm, D. L. (2004). X-tile: a new bio-informatics tool for biomarker assessment and outcomebased cut-point optimization. Clin. Cancer Res. 10, 7252-7259. doi: 10.1158/1078-0432.CCR-04-0713

Cancer Genome Atlas Research, N. (2014). Integrated genomic characterization of papillary thyroid carcinoma. Cell 159, 676-690. doi: 10.1016/j.cell.2014.09.050

Chen, L. L., and Yang, L. (2015). Regulation of circRNA biogenesis. RNA Biol. 12, 381-388. doi: 10.1080/15476286.2015.1020271

Delprat, B., Schaer, D., Roy, S., Wang, J., Puel, J. L., and Geering, K. (2007). FXYD6 is a novel regulator of $\mathrm{Na}, \mathrm{K}$-ATPase expressed in the inner ear. J. Biol. Chem. 282, 7450-7456. doi: 10.1074/jbc.M609872200

Dudekula, D. B., Panda, A. C., Grammatikakis, I., De S, Abdelmohsen, K., and Gorospe, M. (2016). CircInteractome: a web tool for exploring circular RNAs and their interacting proteins and microRNAs. RNA Biol. 13, 34-42. doi: 10.1080/15476286.2015.1128065

Estévez, R., Boettger, T., Stein, V., Birkenhäger, R., Otto, E., Hildebrandt, F., et al. (2001). Barttin is a Cl- channel beta-subunit crucial for renal Cl- reabsorption and inner ear K+ secretion. Nature 414, 558-561. doi: 10.1038/35107099

Ferru, A., Fromont, G., Gibelin, H., Guilhot, J., Savagner, F., Tourani, J. M., et al. (2006). The status of CDKN2A alpha (p16INK4A) and beta (p14ARF) transcripts in thyroid tumour progression. Br. J. Cancer 95, 1670-1677. doi: $10.1038 /$ sj.bjc. 6603479

\section{ACKNOWLEDGMENTS}

We thank Professor Yupei Zhao from Peking Union Medical College Hospital for his support and guidance in this research. This manuscript has been released as a pre-print at Research Square (Wu et al., 2020).

\section{SUPPLEMENTARY MATERIAL}

The Supplementary Material for this article can be found online at: https://www.frontiersin.org/articles/10.3389/fcell.2020. 606327/full\#supplementary-material

Fontana, R., Ranieri, M., La Mantia, G., and Vivo, M. (2019). Dual role of the alternative reading frame ARF protein in Cancer. Biomolecules 9:87. doi: 10.3390/biom 9030087

Girgis, A. H., Iakovlev, V. V., Beheshti, B., Bayani, J., Squire, J. A., Bui, A., et al. (2012). Multilevel whole-genome analysis reveals candidate biomarkers in clear cell renal cell carcinoma. Cancer Res. 72, 5273-5284. doi: 10.1158/0008-5472.CAN-12-0656

Glenn, K. A., Nelson, R. F., Wen, H. M., Mallinger, A. J., and Paulson, H. L. (2008). Diversity in tissue expression, substrate binding, and SCF complex formation for a lectin family of ubiquitin ligases. J. Biol. Chem. 283, 12717-12729. doi: 10.1074/jbc.M709508200

Guan, Y. J., Ma, J. Y., and Song, W. (2019). Identification of circRNA-miRNAmRNA regulatory network in gastric cancer by analysis of microarray data. Cancer Cell Int. 19:183. doi: 10.1186/s12935-019-0905-Z

Han, P. A., Kim, H. S., Cho, S., Fazeli, R., Najafian, A., Khawaja, H., et al. (2016). Association of BRAF V600E mutation and microRNA expression with central lymph node metastases in papillary thyroid cancer: a prospective study from four endocrine surgery centers. Thyroid 26, 532-542. doi: 10.1089/thy. 2015.0378

Haugen, B. R., Alexander, E. K., Bible, K. C., Doherty, G. M., Mandel, S. J., Nikiforov, Y. E., et al. (2016). 2015 American thyroid association management guidelines for adult patients with thyroid nodules and differentiated thyroid cancer: the American thyroid association guidelines task force on thyroid nodules and differentiated thyroid cancer. Thyroid 26, 1-133. doi: 10.1089/thy.2015.0020

Hill, H. A., Qi, X., Jain, P., Nomie, K., Wang, Y., Zhou, S., et al. (2020). Genetic mutations and features of mantle cell lymphoma: a systematic review and meta-analysis. Blood Adv. 4, 2927-2938. doi: 10.1182/bloodadvances. 2019001350

Huang da, W., Sherman, B. T., and Lempicki, R. A. (2009). Systematic and integrative analysis of large gene lists using DAVID bioinformatics resources. Nat. Protoc. 4, 44-57. doi: 10.1038/nprot.2008.211

Humbey, O., Pimkina, J., Zilfou, J. T., Jarnik, M., Dominguez-Brauer, C., Burgess, D. J., et al. (2008). The ARF tumor suppressor can promote the progression of some tumors. Cancer Res. 68, 9608-9613. doi: 10.1158/0008-5472.CAN-08-2263

Kos, A., Dijkema, R., Arnberg, A. C., van der Meide, P. H., and Schellekens, H. (1986). The hepatitis delta (delta) virus possesses a circular RNA. Nature 323, 558-560. doi: 10.1038/323558a0

La Vecchia, C., Malvezzi, M., Bosetti, C., Garavello, W., Bertuccio, P., Levi, F., et al. (2015). Thyroid cancer mortality and incidence: a global overview. Int. J. Cancer 136, 2187-2195. doi: 10.1002/ijc.29251

Lee, Y. K., Park, J. Y., Kang, H. J., and Cho, H. C. (2003). Overexpression of p16INK4A and p14ARF in haematological malignancies. Clin. Lab. Haematol. 25, 233-237. doi: 10.1046/j.1365-2257.2003. 00520.x

Li, X., Tian, Y., Hu, Y., Yang, Z., Zhang, L., and Luo, J. (2018). CircNUP214 sponges miR-145 to promote the expression of ZEB2 in thyroid cancer cells. Biochem. Biophys. Res. Commun. 507, 168-172. doi: 10.1016/j.bbrc.2018. 10.200 
Liu, F., Zhang, J., Qin, L., Yang, Z., Xiong, J., Zhang, Y., et al. (2018). Circular RNA EIF6 (Hsa_circ_0060060) sponges miR-144-3p to promote the cisplatinresistance of human thyroid carcinoma cells by autophagy regulation. Aging 10, 3806-3820. doi: 10.18632/aging.101674

Liu, J., Zhang, X., Yan, M., and Li, H. (2020). Emerging role of circular RNAs in cancer. Front. Oncol. 10:663. doi: 10.3389/fonc.2020.00663

McCleland, M. L., Kallio, M. J., Barrett-Wilt, G. A., Kestner, C. A., Shabanowitz, J., Hunt, D. F., et al. (2004). The vertebrate $\mathrm{Ndc} 80$ complex contains Spc24 and Spc25 homologs, which are required to establish and maintain kinetochore-microtubule attachment. Curr. Biol. 14, 131-137. doi: $10.1016 /$ j.cub.2003.12.058

Montero-Conde, C., Graña-Castro, O., Martín-Serrano, G., Martínez-Montes, Á. M., Zarzuela, E., Muñoz, J., et al. (2020). Hsa-miR-139-5p is a prognostic thyroid cancer marker involved in HNRNPF-mediated alternative splicing. Int. J. Cancer 146, 521-530. doi: 10.1002/ijc.32622

Murakami, T., Sano, F., Huang, Y., Komiya, A., Baba, M., Osada, Y., et al. (2007). Identification and characterization of Birt-Hogg-Dubé associated renal carcinoma. J. Pathol. 211, 524-531. doi: 10.1002/path.2139

Peng, N., Shi, L., Zhang, Q., Hu, Y., Wang, N., and Ye, H. (2017). Microarray profiling of circular RNAs in human papillary thyroid carcinoma. PLoS ONE 12:e0170287. doi: 10.1371/journal.pone.0170287

Qiu, L., Wang, T., Ge, Q., Xu, H., Wu, Y., Tang, Q., et al. (2019). Circular RNA signature in hepatocellular carcinoma. J. Cancer 10, 3361-3372. doi: $10.7150 /$ jca. 31243

Riesco-Eizaguirre, G., Wert-Lamas, L., Perales-Patón, J., Sastre-Perona, A., Fernández, L. P., and Santisteban, P. (2015). The miR-146b-3p/PAX8/NIS regulatory circuit modulates the differentiation phenotype and function of thyroid cells during carcinogenesis. Cancer Res. 75, 4119-4130. doi: 10.1158/0008-5472.CAN-14-3547

Ritchie, M. E., Phipson, B., Wu, D., Hu, Y., Law, C. W., Shi, W., et al. (2015). limma powers differential expression analyses for RNA-sequencing and microarray studies. Nucleic Acids Res. 43:e47. doi: 10.1093/nar/gkv007

Salmena, L., Poliseno, L., Tay, Y., Kats, L., and Pandolfi, P. P. (2011). A ceRNA hypothesis: the Rosetta Stone of a hidden RNA language? Cell 146, 353-358. doi: 10.1016/j.cell.2011.07.014

Sánchez-Aguilera, A., Sánchez-Beato, M., García, J. F., Prieto, I., Pollan, M., and Piris, M. A. (2002). p14(ARF) nuclear overexpression in aggressive Bcell lymphomas is a sensor of malfunction of the common tumor suppressor pathways. Blood 99, 1411-1418. doi: 10.1182/blood.V99.4.1411

Sannigrahi, M. K., Sharma, R., Singh, V., Panda, N. K., Rattan, V., and Khullar, M. (2017). Role of Host miRNA Hsa-miR-139-3p in HPV-16-induced Carcinomas. Clin. Cancer Res. 23, 3884-3895. doi: 10.1158/1078-0432.CCR-16-2936

Sticht, C., De La Torre, C., Parveen, A., and Gretz, N. (2018). miRWalk: an online resource for prediction of microRNA binding sites. PLoS ONE 13:e0206239. doi: 10.1371/journal.pone.0206239

Subramanian, A., Tamayo, P., Mootha, V. K., Mukherjee, S., Ebert, B. L., Gillette, M. A., et al. (2005). Gene set enrichment analysis: a knowledge-based approach for interpreting genome-wide expression profiles. Proc. Natl. Acad. Sci. U.S.A. 102, 15545-15550. doi: 10.1073/pnas.0506580102

Tang, Z., Li, C., Kang, B., Gao, G., Li, C., and Zhang, Z. (2017). GEPIA: a web server for cancer and normal gene expression profiling and interactive analyses. Nucleic Acids Res. 45, W98-W102. doi: 10.1093/nar/gkx247

Wei, J., Wang, J., Gao, X., and Qi, F. (2019). Identification of differentially expressed circRNAs and a novel hsa_circ_0000144 that promote tumor growth in gastric cancer. Cancer Cell Int. 19:268. doi: 10.1186/s12935-0190975-y

Wu, M., Yuan, H., Li, X., Liao, Q., and Liu, Z. (2019). Identification of a five-gene signature and establishment of a prognostic nomogram to predict progression-free interval of papillary thyroid Carcinoma. Front. Endocrinol. 10:790. doi: 10.3389/fendo.2019.00790

Wu, M., Liu, R., Yuan, H., Xu, X., Li, X., and Liu, Z. (2020). Progression risk assessment of post-surgical papillary thyroid carcinoma based on circular RNA-associated competing endogenous RNA mechanisms. Res. Square. doi: $10.21203 /$ rs.3.rs-47878/v1
Yang, Y., Ding, L., Li, Y., and Xuan, C. (2020). Hsa_circ_0039411 promotes tumorigenesis and progression of papillary thyroid cancer by miR-1179/ABCA9 and miR-1205/MTA1 signaling pathways. J. Cell. Physiol. 235, 1321-1329. doi: 10.1002/jcp. 29048

Yao, Y., Chen, X., Yang, H., Chen, W., Qian, Y., Yan, Z., et al. (2019). Hsa_circ_0058124 promotes papillary thyroid cancer tumorigenesis and invasiveness through the NOTCH3/GATAD2A axis. J. Exp. Clin. Cancer Res. 38:318. doi: 10.1186/s13046-019-1321-x

Yin, H., Meng, T., Zhou, L., Chen, H., and Song, D. (2017). SPC24 is critical for anaplastic thyroid cancer progression. Oncotarget 8, 21884-21891. doi: 10.18632/oncotarget.15670

Yonemori, M., Seki, N., Yoshino, H., Matsushita, R., Miyamoto, K., Nakagawa, M., et al. (2016). Dual tumor-suppressors miR-139-5p and miR-139-3p targeting matrix metalloprotease 11 in bladder cancer. Cancer Sci. 107, 1233-1242. doi: $10.1111 /$ cas. 13002

Yoo, J. C., Lim, T., Park, J. S., Hah, Y. S., Park, N., Hong, S. G., et al. (2013). SYT14L, especially its $\mathrm{C} 2$ domain, is involved in regulating melanocyte differentiation. J. Dermatol. Sci 72, 246-251. doi: 10.1016/j.jdermsci.2013.07.010

Yu, C., Zhang, L., Luo, D., Yan, F., Liu, J., Shao, S., et al. (2018). MicroRNA-146b-3p promotes cell metastasis by directly targeting NF2 in human papillary thyroid cancer. Thyroid 28, 1627-1641. doi: 10.1089/thy.2017.0626

Zhang, D., and Xia, J. (2020). Somatic synonymous mutations in regulatory elements contribute to the genetic aetiology of melanoma. BMC Med. Genomics 13(Suppl. 5):43. doi: 10.1186/s12920-020-0685-2

Zhang, S., Zeng, X., Ding, T., Guo, L., Li, Y., Ou, S., et al. (2018). Microarray profile of circular RNAs identifies hsa_circ_0014130 as a new circular RNA biomarker in non-small cell lung cancer. Sci. Rep. 8:2878. doi: 10.1038/s41598-018-21300-5

Zhang, W., Zhang, H., and Zhao, X. (2020). circ_0005273 promotes thyroid carcinoma progression by SOX2 expression. Endocr. Relat. Cancer 27, 11-21. doi: 10.1530/ERC-19-0381

Zhong, Y., Du, Y., Yang, X., Mo, Y., Fan, C., Xiong, F., et al. (2018). Circular RNAs function as ceRNAs to regulate and control human cancer progression. Mol. Cancer 17:79. doi: 10.1186/s12943-018-0827-8

Zhou, J., Pei, Y., Chen, G., Cao, C., Liu, J., Ding, C., et al. (2018). SPC24 Regulates breast cancer progression by PI3K/AKT signaling. Gene 675:272-277. doi: 10.1016/j.gene.2018.07.017

Zhou, J., Yu, Y., Pei, Y., Cao, C., Ding, C., Wang, D., et al. (2017). A potential prognostic biomarker SPC24 promotes tumorigenesis and metastasis in lung cancer. Oncotarget 8, 65469-65480. doi: 10.18632/oncotarget. 18971

Zhu, P., Jin, J., Liao, Y., Li, J., Yu, X. Z., Liao, W., et al. (2015). A novel prognostic biomarker SPC24 up-regulated in hepatocellular carcinoma. Oncotarget 6, 41383-41397. doi: 10.18632/oncotarget.5510

Zhuang, C., Huang, X., Yu, J., and Gui, Y. (2020). Circular RNA hsa_circ_0075828 promotes bladder cancer cell proliferation through activation of CREB1. BMB Rep. 53, 82-87. doi: 10.5483/BMBRep.2020.53. 2.059

Conflict of Interest: The authors declare that the research was conducted in the absence of any commercial or financial relationships that could be construed as a potential conflict of interest.

The reviewer CL declared a shared affiliation with the authors to the handling editor at time of review.

Copyright $\odot 2021 \mathrm{Wu}, \mathrm{Li}$, Han, Liu, Yuan, Xu, Li and Liu. This is an open-access article distributed under the terms of the Creative Commons Attribution License (CC $B Y)$. The use, distribution or reproduction in other forums is permitted, provided the original author(s) and the copyright owner(s) are credited and that the original publication in this journal is cited, in accordance with accepted academic practice. No use, distribution or reproduction is permitted which does not comply with these terms. 